\title{
A Facile Synthesis of PbS-G QDs Nanocomposite as Electrode Material With Enhanced Energy Density for High Performance Supercapattery Application
}

\section{G. Suganya}

Anna University Chennai

M. Arivanandhan

Anna University Chennai

Kalpana Gopalakrishnan ( $\nabla$ g_kalpa@yahoo.com )

Anna University Chennai https://orcid.org/0000-0002-2920-7342

\section{Research Article}

Keywords: Quantum dots, TEM, Supercapattery, Energy density, Power density

Posted Date: December 29th, 2021

DOI: https://doi.org/10.21203/rs.3.rs-1181769/v1

License: (1) (1) This work is licensed under a Creative Commons Attribution 4.0 International License.

Read Full License

Version of Record: A version of this preprint was published at Journal of Inorganic and Organometallic Polymers and Materials on March 12th, 2022. See the published version at https://doi.org/10.1007/s10904-022-02274-4. 


\title{
A facile synthesis of PbS-G QDs nanocomposite as electrode material with enhanced energy density for high performance supercapattery application
}

\author{
G. Suganya, ${ }^{a}$ M. Arivanandhan ${ }^{\mathrm{b}}$ and G. Kalpana ${ }^{\mathrm{a} *}$ \\ aDepartment of Physics, Anna University, Chennai - 600025 \\ ${ }^{\mathrm{b}}$ Centre for Nanoscience and Technology, Anna University, Chennai - 600025
}

\begin{abstract}
Bare $\mathrm{PbS}$ QDs and $\mathrm{PbS}$-GQDs nanocomposite were prepared by chemical methods for supercapattery application and characterized by suitable analytical techniques confirming the formation of PbS-GQDs nanocomposite. The electrochemical performance of the fabricated electrodes showed that the PbS-GQDs nanocomposite exhibited high specific capacity, energy and power densities of $577.94 \mathrm{C} \mathrm{g}^{-1}, 166.45 \mathrm{Wh} \mathrm{kg}^{-1}$ and $576.01 \mathrm{~W} \mathrm{~kg}^{-1}$ respectively at $2 \mathrm{~A} \mathrm{~g}^{-1}$ compared to that of bare PbS QDs. The enhanced electrochemical performance of PbS-GQDs can be associated with the conductive platform provided by synergistic effect of GQDs. The nonlinearity in charge and discharge curves confirms the supercapattery behaviour of the nanocomposite. Also, PbS-G QDs nanocomposite electrode showed highly cyclic stability compared to bare PbS QDs after 5000 cycles. The results emphasize the potential of PbS-G QDs nanocomposite as a stable active electrode material for energy storage application.
\end{abstract}

Keywords: Quantum dots; TEM; Supercapattery; Energy density; Power density

*Corresponding author:

Dr.G.Kalpana,

Professor,

Department of Physics,

Anna University, Chennai, India

Email :g_kalpa@yahoo.com 


\section{Introduction}

The supercapattery (batteries and supercapacitor) combines the high-power capability of usual rechargeable batteries with excellent energy density of energy storage devices [1,2]. In particular, developing new materials and technology-based innovations in design of structures are being increasingly recognised and utilised to develop rechargeable batteries to deliver high energy density and supercapacitor to deliver high power capability, short charging time and long cyclic efficiency [3, 4]. The energy storage research has seen significant progress in both rechargeable battery and supercapacitor during the least two decades. The supercapacitors and batteries are being observed as the two most encouraging electrochemical applications [5-7]. On the basis of electrochemical mechanism, supercapacitors are generally classified into electrical double-layer supercapacitors (EDLCs) and pseudo-capacitors. EDLCs capacitive process is governed by aggregation of charge (non-Faradaic) at the electrolyte/electrode interface and pseudocapacitive charge was diffusion-controlled Faradaic redox (battery-type) reaction [8-10]. Generally, there are two different operates, the battery operates based on cation based redox reactions at solid state electrode materials whereas supercapacitor operates based on electrolyte ions of adsorption/desorption at electrode/electrolyte interfaces in the electrochemical energy storage process [11]. Both high specific capacity with high power density and energy density characteristics is achieved in one device named as supercapattery. The behaviour of supercapattery is similar to that of an electrochemical cell with greater performance, high power capability with higher energy density, longer charge-discharge durability and long lifetime [12].

Generally, metal sulphides based on the energy storage device such as CuS [13], CoS [14], $\mathrm{SnS}$ [15], CuS-PbS[16], NiS [17], and $\mathrm{ZnS} \mathrm{[18]} \mathrm{are} \mathrm{redox-active} \mathrm{and} \mathrm{attractive} \mathrm{working} \mathrm{electrode}$ materials for achieving high supercapacitor performance at low cost methods. Nanostructuring the material is the promising way to develop the electrochemical properties due to its enhanced surface area. However, there is little information on the synthesis of PbS QDs materials as an electrode for high performance electrochemical energy storage application. Lead sulphide is the chalcogenide group semiconductor having the direct band gap $(0.41 \mathrm{eV})$ and Bohr radius of about $18 \mathrm{~nm}$ [19]. $\mathrm{PbS}$ QDs are promising semiconductors and active material used in different applications such as optical fiber, energy conversion, solar energy harvesting and energy storage devices [20-22]. The performance of supercapacitor materials depends on multiple parameters, such as surface morphology, adhesion on to the substrate, reaction temperature, and nanostructure properties of the active electrodes being affected by the particle size distribution, surface area, material thickness, transport/diffusion paths, and accessibility of redox electrolyte/electrode. Carbon based 
nanocomposite materials have high surface area and electrical conductivity and due to these properties, it is a promising electrode material for energy storage applications. Recently graphenebased metal oxides such as G-NiO,[23] G- $\mathrm{MnO}_{2}$ [24], G-ZnO [25], G- $\mathrm{V}_{2} \mathrm{O}_{5}$ [26], have been shown to be effective for enhancing the specific capacitance than their individual performance in supercapacitors because of the synergistic effect between the composite materials. Apart from the transition metal oxides, polymer composites have been tested for electrochemical capacitors and Liion batteries due to their good stability [27, 28]. More recently, CNT/CuS [29], CNT/CoS [30] and $\mathrm{Sn} / \mathrm{CNT}$ [31] composite electrodes with a maximum specific capacitance of 122-89 F/g, 1120 F/g and $334-76 \mathrm{~F} / \mathrm{g}$ respectively. However, there is no report on the electrochemical properties of lead sulphide - graphene quantum dots (PbS-G QDs) nanocomposite.

Therefore, in the present work, a cost effective, facile strategy was developed for the design, fabrication and electrochemical testing of PbS-G QDs nanocomposite on a nickel foam substrate using chemical bath (CBD) method for supercapattery applications. The bare PbS QDs, GQDs and PbS-G QDs nanocomposite were synthesized by chemical bath method, ultrasonication method and a simple solvothermal method respectively using low-cost precursor's materials. The structural, optical absorption, band gap, morphological, particle size and specific surface area characterizations of the prepared samples were done and its electrochemical performance was evaluated for energy storage application. The bare $\mathrm{PbS}$ QDs and PbS-G QDs nanocomposite electrodes were analyzed by cyclic voltammetry (CV), galvanostatic charge and discharge (GCD) and electrochemical impedance spectroscopy (EIS) at $2 \mathrm{M} \mathrm{KOH}$ electrolyte ionic solution. The PbS-G QDs nanocomposite electrode material exhibits a high specific capacity, high energy density with power density compared to bare PbS QDs and exhibited $\sim 97.4 \%$ stable capacity retention at 5000 cycles.

\section{Experimental Methods}

\subsection{Materials}

Graphite powder, potassium permanganate $\left(\mathrm{KMnO}_{4}\right)$, hydrogen peroxide $\left(\mathrm{H}_{2} \mathrm{O}_{2}\right)$, sulphuric acid $\left(\mathrm{H}_{2} \mathrm{SO}_{4}\right)$, sodium hydroxide $(\mathrm{NaOH})$, SDS (sodium dodecyl sulfate), CTAB (cetyltrimethyl ammonium bromide), EDTA (ethylene diamine tetraacetic acid), Lead nitrate, Thiourea Ethylene glycol and $\mathrm{NaOH}$ pellets were purchased from Sigma Aldrich. 


\subsection{Synthesis methods of bare PbS QDs, GQDs and PbS-G QDs nanocomposite}

In the first half of the synthesis process, bare $\mathrm{PbS}$ QDs were synthesized by simple chemical bath method [32]. In a typical synthesis procedure, SDS (0.5 g), CTAB (0.5 g), and EDTA (2 g) were dissolved in $100 \mathrm{~mL}$ deionized water (DIW) at $80{ }^{\circ} \mathrm{C}$ under constant stirring condition. The $\mathrm{pH}$ of the solution was increased to 14 by slowly adding $\mathrm{NaOH}$ solution. Next, lead (II) nitrate (2 g) dissolved in $10 \mathrm{~mL}$, DIW was injected into the precursor solution under continuous stirring condition. After that, thiourea $(0.5 \mathrm{~g})$ dissolved in $15 \mathrm{~mL}$, DIW solution was drop wise introduced into the system for a duration of about 30 minutes with continuous vigorous stirring. The continued vigorous stirring for another $1 \mathrm{~h}$ during which the solution turned into brown colour indicating the formation of $\mathrm{PbS}$ QDs. The final solution was cooled at room temperature, washed four times with DIW and ethanol by centrifugation. Finally, the sample was dried at vacuum oven and the particles were collected for further analysis.

In the second half, Graphene oxide (GO) was synthesized by modified Hummer's method [33, 34]. Next, GQDs were prepared by chemical cutting of graphene oxide with small quantity of strong acid using vertical probe ultrasonication technique [35]. GO (1 g) was dissolved in $200 \mathrm{ml}$ of DIW and ultrasonicated (Sonics Vibracell, $1500 \mathrm{~W}, 20 \mathrm{kHz}, 35^{\circ}$ C) for 15 minutes. Next, this solution was mixed with $50 \mathrm{ml}$ of concentrated sulphuric acid $\left(\mathrm{H}_{2} \mathrm{SO}_{4}\right)$ and once again ultrasonicated for 15 mins. Finally, the solution was cooled to room temperature, centrifuged at 10,000 rpm and washed with DIW and ethanol several times. After removing the acids, the chocolate brown supernatant was collected and dried in vacuum oven as the final GQDs product.

Next PbS-Graphene QDs nanocomposite was prepared by solvothermal method [36]. In brief the lab synthesised PbS QDs ( $1 \mathrm{~g})$ was dispersed in $60 \mathrm{~mL}$ of ethylene glycol by sonication for $1 \mathrm{~h}$ to form a homogeneous mixture, followed by $0.2 \mathrm{~g}$ of synthesised GQDs dissolved separately in ethylene glycol and added to the above PbS QDs dispersed solution under continuous stirring. Then $0.1 \mathrm{M} \mathrm{NaOH}$ was diluted in $5 \mathrm{~mL}$ of DIW and added to the reaction mixture and continuously stirred for $30 \mathrm{~min}$. After complete dispersion, the solution was transferred into autoclave (100 $\mathrm{mL})$ and then kept at $180{ }^{\circ} \mathrm{C}$ for $48 \mathrm{~h}$ in an oven. The obtained final solution was washed with DIW and ethanol three times and dried at $80{ }^{\circ} \mathrm{C}$ for $10 \mathrm{~h}$. The collected black powder of PbS-G QDs nanocomposite was obtained. 


\subsection{Characterization techniques}

The powder X-ray diffraction (XRD) pattern of the synthesized samples were recorded by Rigaku smart X-ray diffractometer equipped with $\mathrm{Cu}-\mathrm{K}_{\alpha}$ radiation $(\lambda=1.54178 \AA)$ to determine the crystal structure and lattice parameters. Optical properties and energy gap were analyzed by UV-vis DRS spectrophotometer. The surface area, pore size and their distribution were analyzed by Brunauer-Emmett-Teller (BET). The formation of PbS-G QDs nanocomposite was further confirmed by Raman analysis. The particle size and crystalline nature of the samples were investigated by TEM and EDS elemental analysis.

\subsection{Electrochemical studies}

The electrochemical tests were analysed using CHI600C electrochemical workstation with three-electrode cell system consisting of $\mathrm{Ag} / \mathrm{AgCl}$, Nickel foam and platinum wire as reference, working and counter electrodes respectively.

\subsection{Electrode Fabrication}

Two different working electrodes with bare $\mathrm{PbS}$ QDs and PbS-G QDs nanocomposite were fabricated separately for comparative studies as follows: Mixture of PbS QDs or PbS-G QDs (80 wt \%) with carbon black (10 wt \%), polyvinylidene fluoride (PVDF) (10 wt \%) were mixed with appropriate amount of N-Methylpyrrolidone (NMP) solvent to make homogeneous mixture. The mixture was coated onto $\mathrm{Ni}$ foam $\left(1 \times 1 \mathrm{~cm}^{2}\right)$ electrode and dried at 100 ${ }^{\circ} \mathrm{C}$ for the solvent to evaporate. The active materials on the $\mathrm{Ni}$ foam is $1 \mathrm{mg} \mathrm{cm}{ }^{-2}$ in both the cases. The electrochemical performance of the bare PbS QDs and PbS-G QDs nanocomposite electrode materials were analysed using GCD, CV and EIS techniques in $2 \mathrm{M} \mathrm{KOH}$ electrolyte in the potential window of $0 \mathrm{~V}$ to $0.5 \mathrm{~V}$.

\section{Results and discussion}

\subsection{X-ray diffraction (XRD) analysis}

The XRD peaks of the synthesized bare PbS QDs and GQD's are shown in Fig.1a. In the case of bare $\mathrm{PbS}$ QDs noticeable diffraction peaks can be observed corresponding to $2 \theta$ values of $25.9^{\circ}$, $30.0^{\circ}, 43.0^{\circ}, 50.9^{\circ}, 53.3^{\circ}, 62.4^{\circ}, 68.8^{\circ}, 70.8^{\circ}$ and $78.8^{\circ}$. These prominent peaks correspond to $(h k$ 
l) planes of (111), (200), (220), (311), (222), (400), (331), (420) and (422) and the pattern is well matched with JCPDS data (No.05-0592). The sharp peaks of the diffraction pattern illustrate high crystalline nature of the sample and the cubic structure of bare PbS QDs is confirmed with lattice constant $\mathrm{a}=5.94 \AA$. In the case of GQD's (Fig. 1a.) the peaks are very weak indicating that GQDs are thinner and ultra-fine size. This is because some layers of graphene were present as a result of $\pi$ $\pi$ stacking. In the case of PbS-G QDs nanocomposite, Fig. $1 \mathrm{~b}$, the peaks at $2 \theta$ values of $23^{\circ}, 31^{\circ}$ and $34^{\circ}$, show the presence of both GQDs and PbS QDs. The average crystallite size of PbS-G QDs nanocomposite and bare PbS QDs, were estimated using Debye Scherrer formula [37] and found to be in the range of $1.4 \mathrm{~nm}$ and $10.6 \mathrm{~nm}$, respectively.
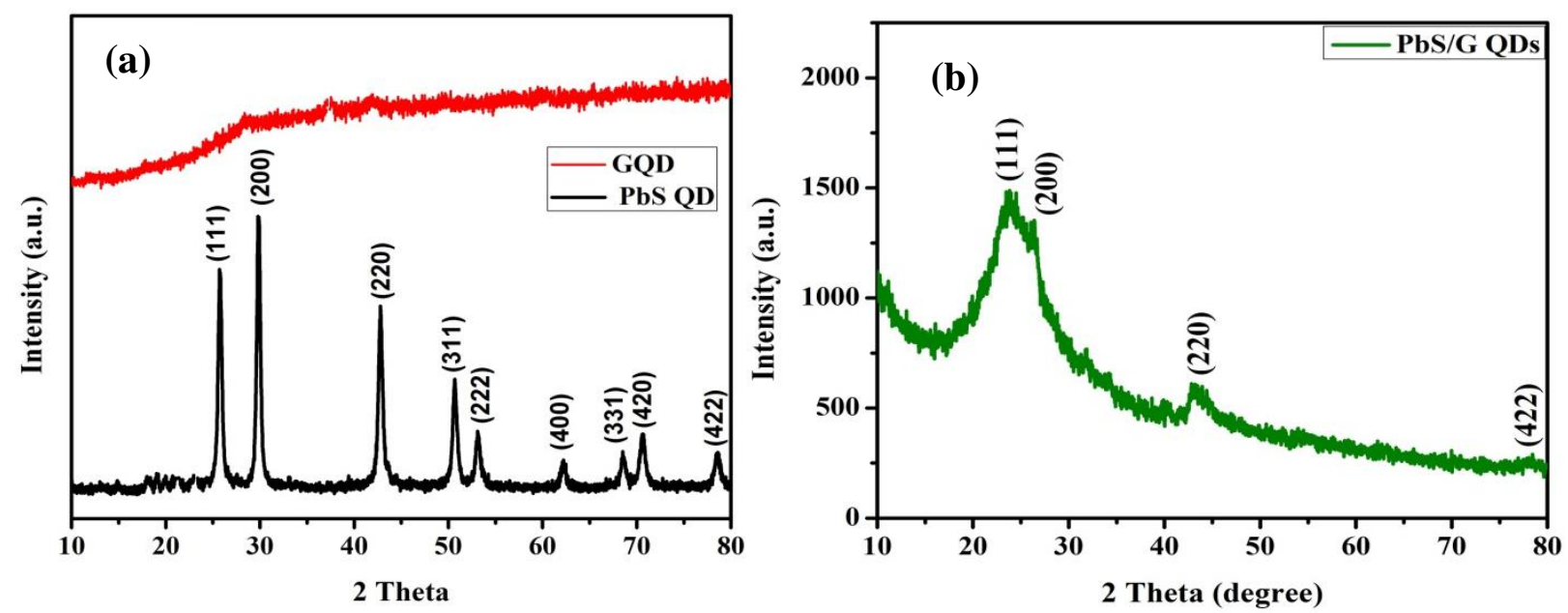

Fig.1. XRD patterns of the mesoporous (a) bare PbS QDs and GQDs, (b) PbS-G QDs nanocomposite.

\subsection{UV-vis diffuse reflectance spectroscopy (UV-vis DRS)}

Optical properties of synthesized bare PbS QDs and PbS-G QDs nanocomposite were analyzed using UV-vis optical absorbance spectra and shown in Fig. 2(a, b). The bare PbS QDs and PbS-G QDs nanocomposite show absorption peak in the UV region at $\sim 480 \mathrm{~nm}$ and $\sim 380$ $\mathrm{nm}$ respectively. The optical absorption peak of PbS-G QDs nanocomposite is slightly shifted to blue region from $\sim 480$ to $\sim 380 \mathrm{~nm}$. It is obvious that the addition of graphene is responsible for the shift in band gap. Different absorption behaviour is observed for bare PbS QDs and PbS-G QDs nanocomposite. The energy gap was calculated using equation (1) from UV-vis DRS spectrum [38]

$$
(\alpha h v)^{\mathrm{n}}=\mathrm{A}\left(\mathrm{h} v-\mathrm{E}_{\mathrm{g}}\right)^{\mathrm{n}}
$$


To find the energy gap, a plot between $(\alpha h v)^{\mathrm{n}}$ vs. (hv) was drawn, where $\alpha$ is the absorption coefficient of the material, hv is the photon energy, A is energy independent constant, $\mathrm{E}_{\mathrm{g}}$ is the band gap, and $\mathrm{n}$ is either 2 and $1 / 2$ for indirect and direct transitions respectively. Thus, a plot of a straight line whose intercept on the energy axis gives the energy gap. According to the above equation, based on the indirect and direct transition, the energy gaps of as-obtained bare PbS QDs is $1.25 \mathrm{eV}$ and PbS-G QDs nanocomposite is $1.95 \mathrm{eV}$ which are shown in inset Fig. 2 (a, b).
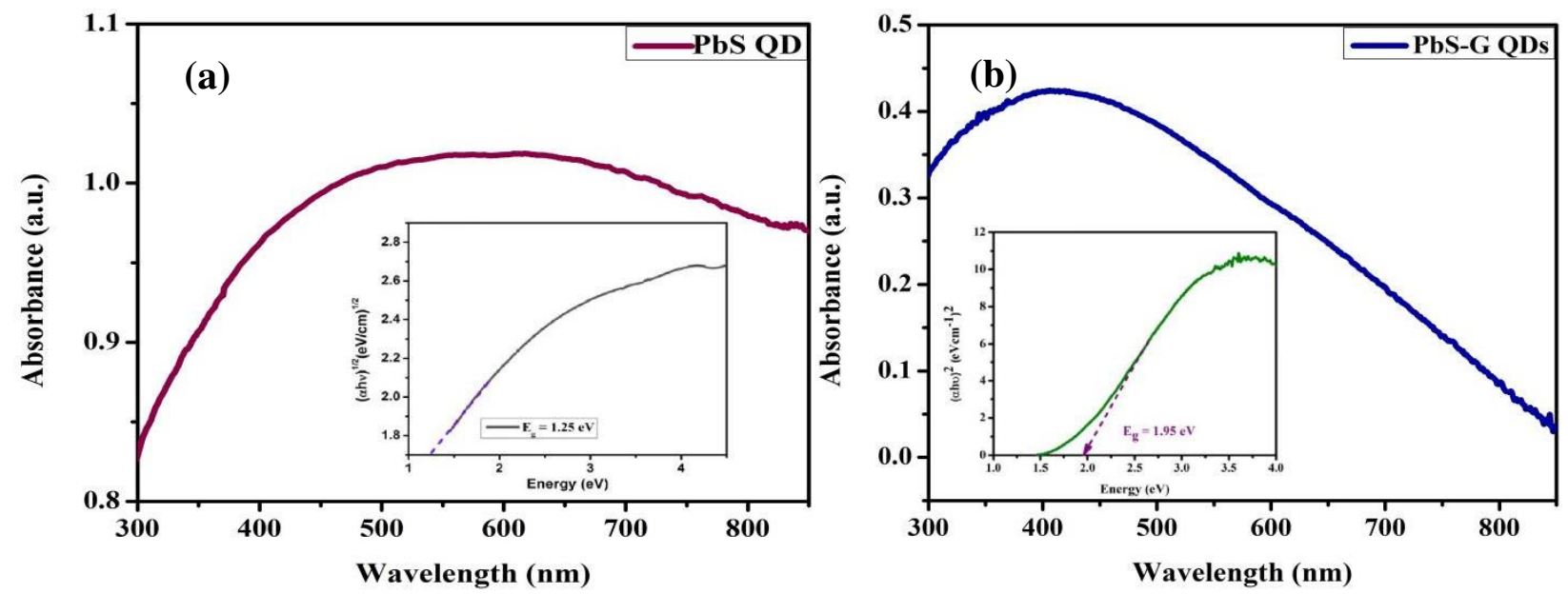

Fig. 2. UV-vis DRS spectrum and optical energy gap diagram (inset picture) of (a) bare PbS QDs and (b) PbS-G QDs nanocomposite.

\subsection{Brunauer-Emmett-Teller (BET)}

The surface properties and the results of the porosity analysis of bare PbS QDs and PbS-G QDs nanocomposite are shown in Fig. $3(\mathrm{a}, \mathrm{b})$ and the corresponding textural parameters are displayed in Table 1. The nitrogen adsorption/desorption isotherm result of the bare PbS QDs and PbS-G QDs nanocomposite clearly exhibit a similar type II curve. The pore size calculated using the BJH method is about $18.088 \mathrm{~nm}$. The BET surface area of the bare PbS QDs is $66.031 \mathrm{~m}^{2} \mathrm{~g}^{-1}$. According to the hysteresis loop at $\mathrm{P} / \mathrm{P}_{0}$ of about $0.45-0.98$, the nitrogen adsorption/desorption isotherms show that the PbS-G QDs nanocomposite exhibit a similar type IV isotherm curve. In other words, the PbS-G QDs nanocomposite exist with a mesoporous structure. Due to the mesoporous structure, the PbS-G QDs nanocomposite show a nanopore size of $11.299 \mathrm{~nm}$ and a very large surface area of $107.74 \mathrm{~m}^{2} \mathrm{~g}^{-1}$ as measured from BET analysis. Besides, the total pore volume of PbS-G QDs nanocomposite is $24.753 \mathrm{~cm}^{3} \mathrm{~g}^{-1}$, which is higher than the total pore volume of bare PbS QDs $\left(15.171 \mathrm{~cm}^{3} \mathrm{~g}^{-1}\right)$. The PbS-G QDs nanocomposite can be expected to have a high electrochemical activity with an increase in the surface area and decrease in pore size, as well as large total pore volume, 

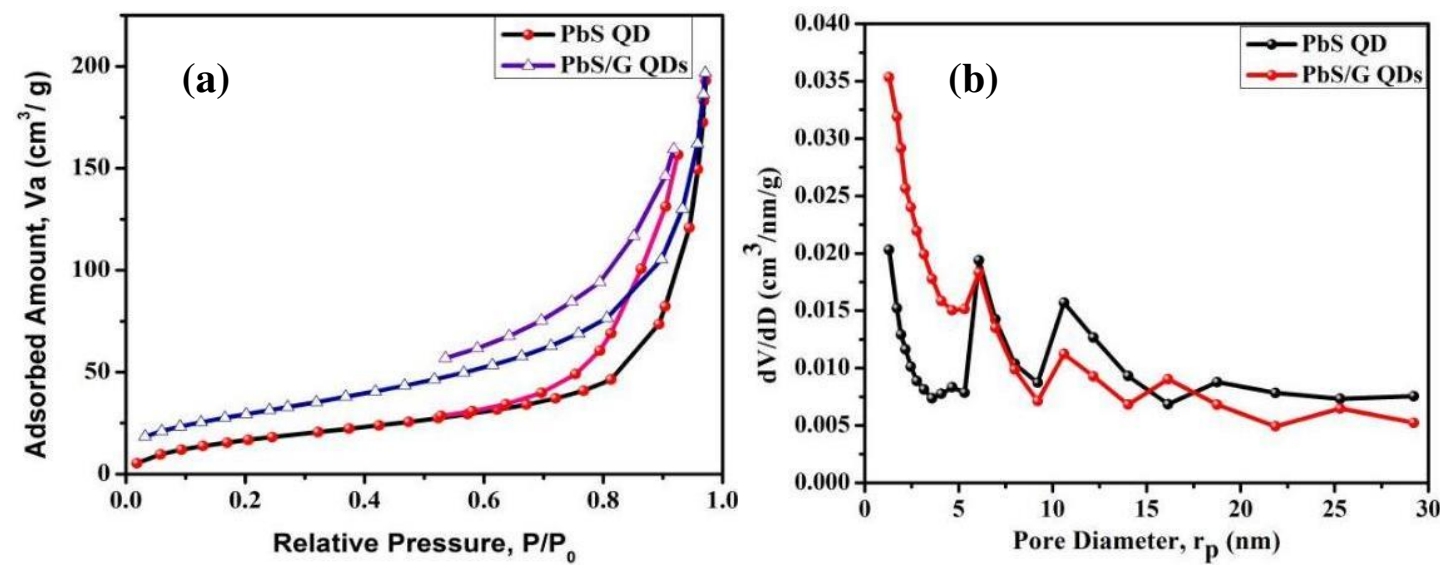

Fig. 3 (a) The nitrogen adsorption/desorption isotherms and (b) pore size distributions for the bare PbS QDs and PbS-G QDs nanocomposites.

Table 1 Micropore structure parameters of the bare PbS QDs and PbS/G QDs nanocomposite.

\begin{tabular}{|l|l|l|l|}
\hline Sample & $\begin{array}{l}\text { BET } \\
\left(\mathbf{m}^{\mathbf{2}} \mathbf{g}\right.\end{array}$ & $\begin{array}{l}\text { Total pore volume } \\
\left(\mathbf{c m}^{\mathbf{1}} \mathbf{g}^{-\mathbf{1}}\right)\end{array}$ & $\begin{array}{l}\text { Average pore } \\
\text { size (nm) }\end{array}$ \\
\hline Bare PbS QDs & 66.031 & 15.171 & 18.088 \\
\hline $\begin{array}{l}\text { PbS-G QDs } \\
\text { nanocomposite }\end{array}$ & 107.74 & 24.753 & 11.299 \\
\hline
\end{tabular}

\subsection{Micro-Raman scattering analysis}

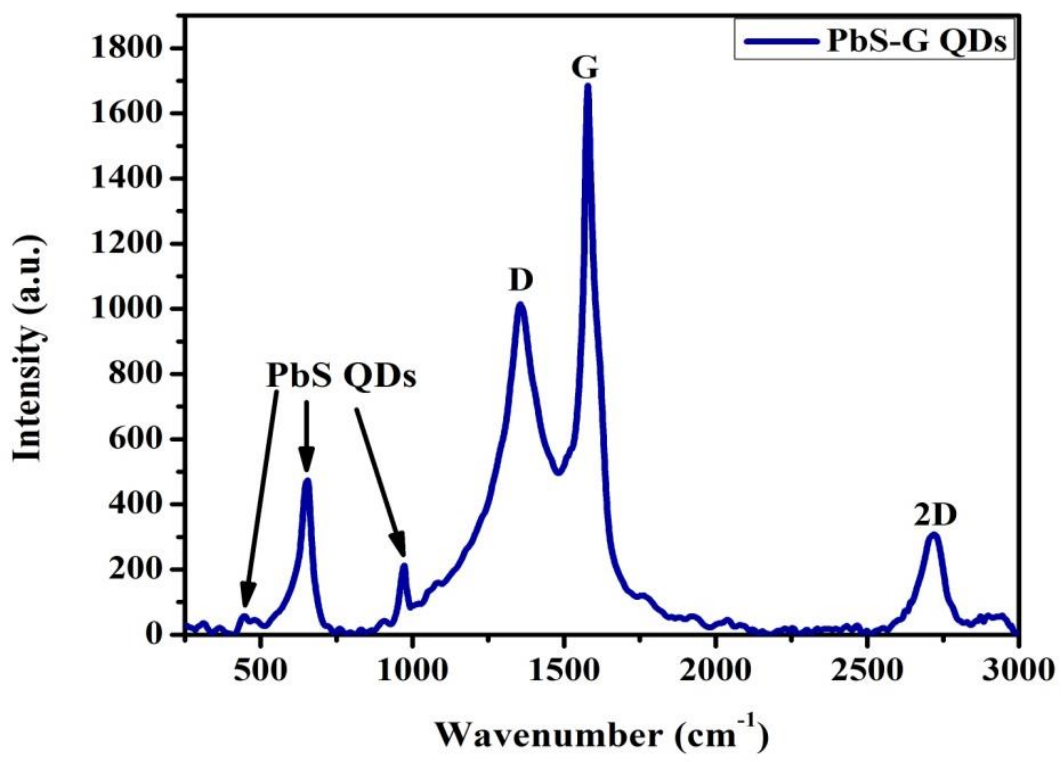

Fig. 4. Raman spectrum of PbS-G QDs nanocomposite 
Raman analysis was performed to examine the formation of PbS-G QDs nanocomposites [39]. Fig. 4 shows the Raman spectrum of the PbS-G QDs nanocomposite in the wavenumber range between $100 \mathrm{~cm}^{-1}$ and $3100 \mathrm{~cm}^{-1}$. In the Raman spectrum of the PbS-G QDs nanocomposite, three intensive Raman peaks are observed at $1355.7 \mathrm{~cm}^{-1}, 1580.6 \mathrm{~cm}^{-1}$ and $2719.1 \mathrm{~cm}^{-1}$, corresponding to $\mathrm{D}, \mathrm{G}$ and $2 \mathrm{D}$ bands of GQDs in the composite. The peak related to $\mathrm{D}$ band is a disordered band arising from the $s p^{2}$ hybridized carbon, while the $\mathrm{G}$ band shows the graphitic carbon. The broad peak observed at $2719.1 \mathrm{~cm}^{-1}$ corresponds to the $2 \mathrm{D}$ band of GQDs. It arises from relaxation in the selection rules caused by phonon scattering at boundaries and defects in GQDs. Based on the Raman spectrum, one can find that the intensity ratio of $\mathrm{D}$ band to $\mathrm{G}$ band $\left(\mathrm{I}_{\mathrm{D}} / \mathrm{I}_{\mathrm{G}}\right)$ is about 1.15 . The intensity ratio $\mathrm{I}_{\mathrm{D}} / \mathrm{I}_{\mathrm{G}}$ is found to vary inversely with the cluster size $\mathrm{L}_{\mathrm{a}}$ according to the following relation (2) [40]

$$
\mathrm{I}_{\mathrm{D}} / \mathrm{I}_{\mathrm{G}=\mathrm{C}}(\lambda) / \mathrm{L}_{\mathrm{a}}
$$

where $C(\lambda)$ is an empirical constant that depends on the excitation laser energy $C(\lambda=632.8 \mathrm{~nm})$. According to this relation, the average size $\left(\mathrm{L}_{\mathrm{a}}\right)$ of GQDs is about $4.3 \mathrm{~nm}$. Further the three peaks observed at 448.6, 650.9 and $968.5 \mathrm{~cm}^{-1}$, are associated with $\mathrm{PbS}$ QDs [41]. The Raman mode observed at $448.6 \mathrm{~cm}^{-1}$ corresponds to the $1 \mathrm{LO}$ vibrational phonon mode of the $\mathrm{PbS} \mathrm{QD}$. The peaks at $650.9 \mathrm{~cm}^{-1}$ and $968.5 \mathrm{~cm}^{-1}$ correspond to interface phonon mode (2LO) and high phonon frequency mode (3LO) respectively. Also the intensity of the peaks depends on the QD size. The Raman peaks corresponding to $\mathrm{PbS}$ QDs and GQDs confirmed the formation of PbS-G QDs nanocomposites.

\subsection{TEM analysis}

The surface morphology of bare PbS QDs and PbS-G QDs nanocomposite are obtained from TEM and the images are shown in Fig. 5(a,b) and 5(d,e). TEM images show distinctly visible and uniformly distributed narrow spherical dot like structures of PbS QDs, which is portrayed in Fig. 5 (a,b). The TEM image of PbS-G QDs nanocomposite under low magnification (Fig. 5d) shows that $\mathrm{PbS}$ QDs are decorated onto GQDs, whereas high magnification image (Fig. 5c) shows dense PbS QDs being uniformly anchored onto GQDs surface. As shown in the figures, the PbS QDs and PbSG QDs particle sizes are in the range of $5.0 \mathrm{~nm}$. Fig. 5c and 5f shows selected area electron diffraction (SAED) patterns of bare PbS QDs and PbS-G QDs nanocomposites. The circular pattern with clear dots illustrates the polycrystalline nature of both the samples. The EDS analyses of synthesized PbS-G QDs nanocomposite establish the chemical composition of the synthesized product and the results are shown in Fig. 6(a), which depicts the presence of carbon (C) 85.77\%, 
lead $(\mathrm{Pb})$ 12.59\% and sulphide $(\mathrm{S})$ 1.64\%. The densely agglomerated region of the PbS-G QDs nanocomposite from EDS mapping is shown in Fig. 6 (b,c,d,e,f). The synthesized PbS-G QDs nanocomposite is free from other elemental impurities.
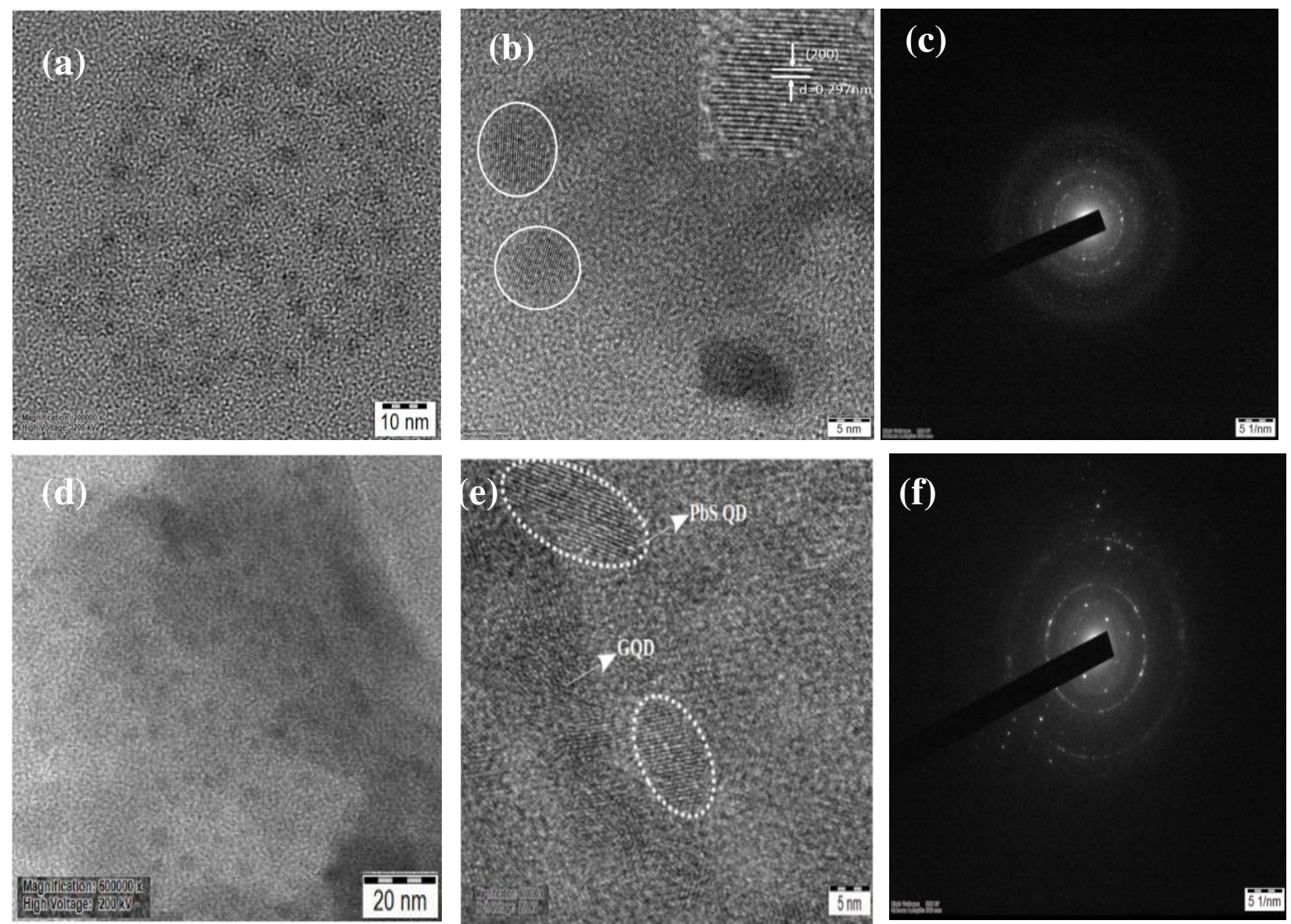

Fig.5. HRTEM images of (a \& b) bare PbS QD, (d \& e) PbS-G QDs nanocomposite and SAED patterns of (c) bare PbS QD and (f) PbS-G QDs.

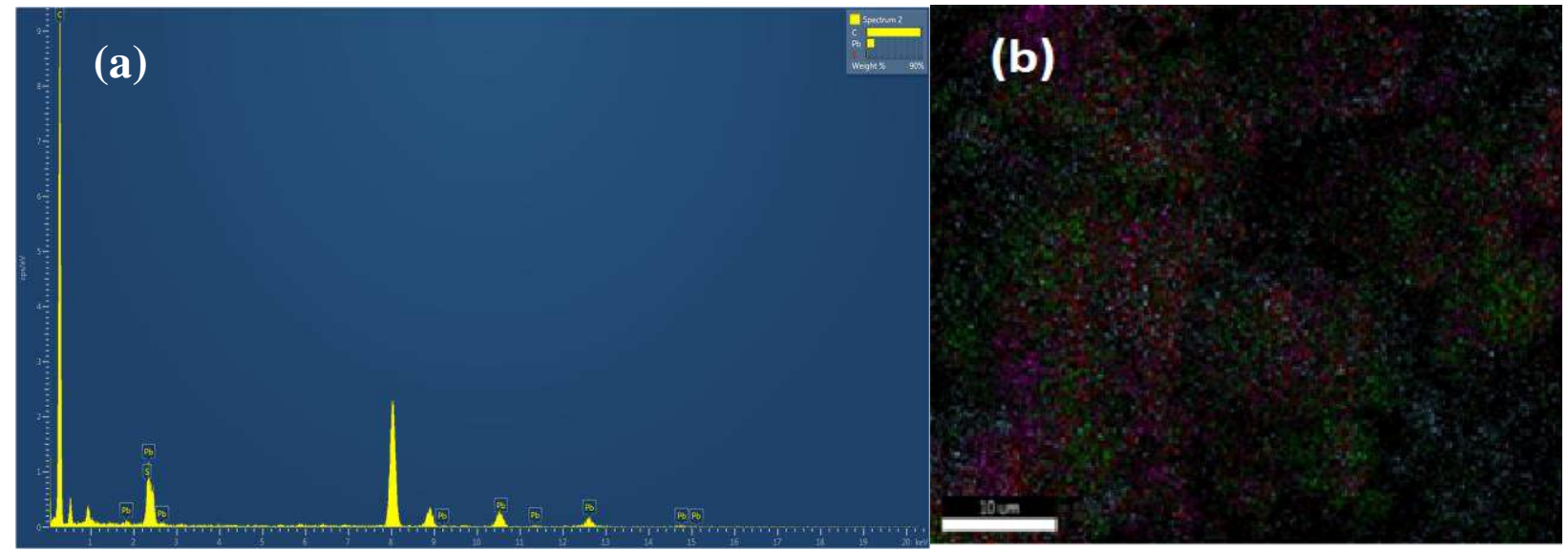




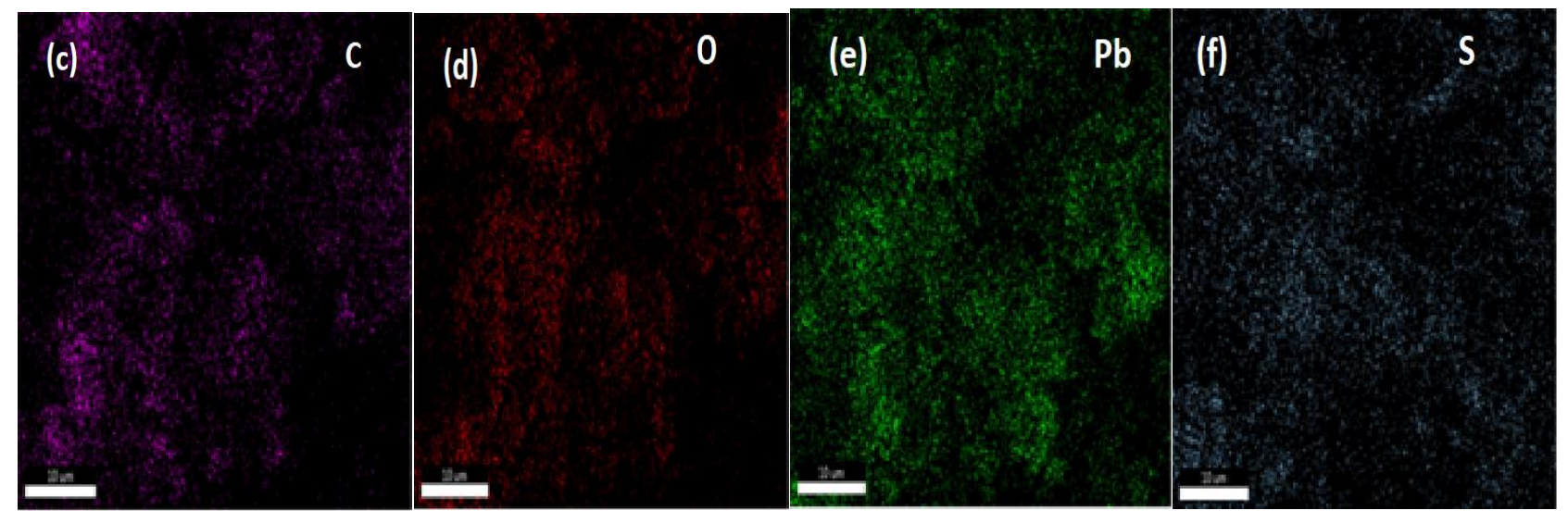

Fig. 6. (a) EDX spectrum and mapping images of (b) PbS-G QDs nanocomposite, (c) C, (d) O, (e) $\mathrm{Pb}$ and (f) $\mathrm{S}$ in the nanocomposite.

\subsection{Electrochemical studies}

\subsubsection{Electrochemical studies of bare PbS QDs and PbS-G QDs nanocomposite}

The cyclic voltammetry (CV) curves of bare PbS QDs and PbS-G QDs nanocomposite for various scan rates $\left(5-100 \mathrm{mV} \mathrm{s}^{-1}\right)$ in the potential window of 0.0 to $0.5 \mathrm{~V}$ are shown in Fig. 7 (a, b). The electrochemical properties of the electrode materials were measured in 2 $\mathrm{M} \mathrm{KOH}$ electrolyte solution at room temperature. It is clearly seen from Fig. 7, that both the materials exhibit redox $\mathrm{CV}$ curves, indicating that the charge storage mechanism is due to pseudocapacitance behaviour [42]. The fast and irreversible Faradic redox (oxidation/reduction) reaction of $\mathrm{Pb}$ present in both the materials in responsible for this behaviour [43]. While making a comparison between the $\mathrm{CV}$ curves of bare $\mathrm{PbS}$ QDs and $\mathrm{PbS}-\mathrm{G}$ QDs nanocomposite, later possesses maximum area under the curve, revealing its improved capacitance than the other. The $\mathrm{K}^{+}$ ions present in the electrolyte also involves in the Faradaic reaction. Moreover, $\mathrm{PbS}$ materials hold the battery type behaviour [44], and hence these two materials can be used for supercapattery application. The CV curves almost remain same in shape even at high scan rate, indicating high-rate capability of the prepared electrode materials. The proposed possible oxidation-reduction process concerning the intercalation/deintercalation of alkaline medium $\left(\mathrm{K}^{+}\right)$into $\mathrm{PbS}$ QDs/ PbS-G QDs could be given via the following reactions $[45,46]$ 
$(\mathrm{PbS} \mathrm{QD})_{\text {surface }}+\mathrm{K}^{+}+\mathrm{e}^{-} \leftrightarrow[\mathrm{KPbS} \mathrm{QD}]_{\text {surface }}$

$\mathrm{PbS} \mathrm{QD}+\mathrm{K}^{+}+\mathrm{e}^{-} \leftrightarrow[\mathrm{PbS} \mathrm{QD}] \mathrm{K}$

and

$(\mathrm{PbS}-\mathrm{G} \mathrm{QDs})_{\text {surface }}+\mathrm{K}^{+}+\mathrm{e}^{-} \leftrightarrow[\mathrm{KPbS}-\mathrm{G} \mathrm{QDs}]_{\text {surface }}$

$\mathrm{PbS}-\mathrm{G}$ QDs $+\mathrm{K}^{+}+\mathrm{e}^{-} \leftrightarrow[\mathrm{PbS}-\mathrm{G}$ QDs] K
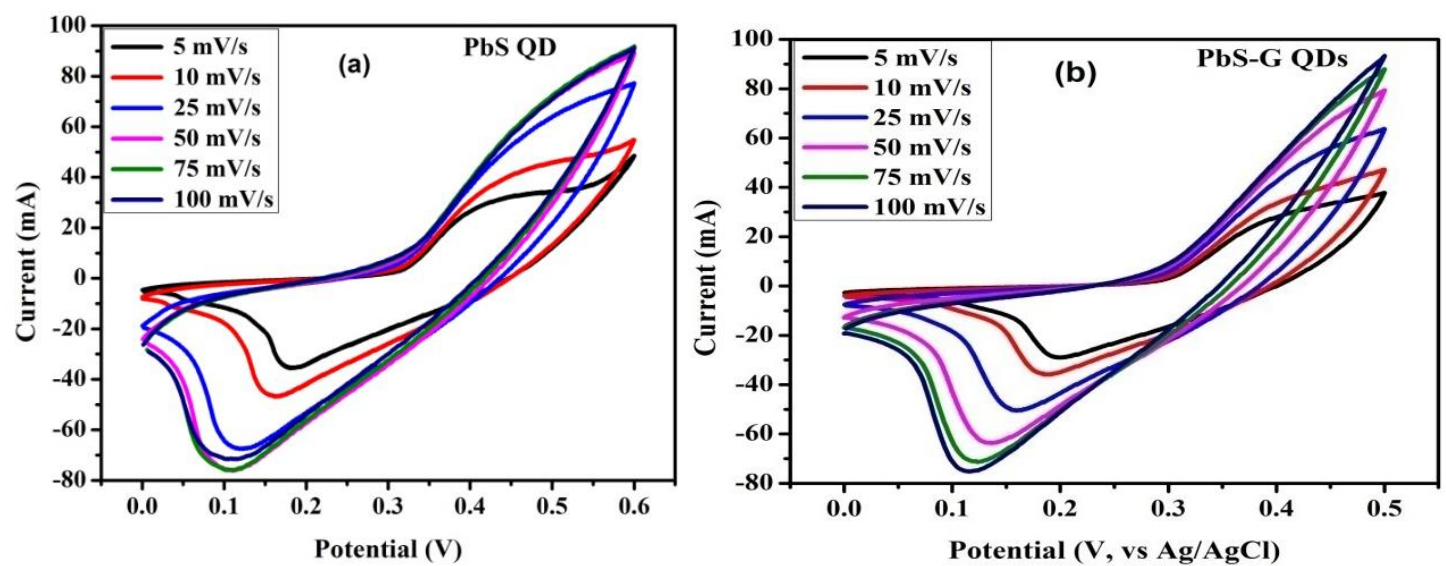

Fig. 7. Cyclic voltammograms at different scan rates (a) bare $\mathrm{PbS}$ QDs and (b) PbS-G QDs nanocomposite

The introduction of GQDs has significantly reduced the particle aggregation leading to exposure of more active sites of electrode material for the redox reactions. In addition, the porous structure and large surface area of GQDs provide rapid charge transfer kinetics, which aid Faradaic reactions [47]. Moreover, in the case of PbS-G QDs nanocomposite (Fig. 7b), the distortion in the CV curve and shift in redox peaks were significant compared to bare PbS QDs. This reveals the improved electrochemical reversible behaviour of the nanocomposite. The observed significant increase in redox peak is due to the synergistic effect of GQDs which provides extended channels for electron transfer during redox process. The excellent electronic conductivity and high surface area of GQDs assembly enhanced the electrochemical performance of the PbS-G QDs nanocomposite [48]. Therefore, the decoration of $\mathrm{PbS}$ QDs on GQDs further improves its conductivity and capacitive performance. The specific capacity (Qs) of the electrode materials was calculated from the CV plot at different scan rates using the following equation (2) [49]

$$
Q_{S}=\frac{1}{v m} \int_{v i}^{v f} I \times V d V \quad \quad \mathrm{C} \mathrm{g}^{-1}
$$

where, $\mathrm{v}$ is the scan rate $\left(\mathrm{mV} \mathrm{s}^{-1}\right), \mathrm{m}$ is the mass of the active electrode materials $(\mathrm{g})$ and the anodic peak area under the $\mathrm{CV}$ curve is equivalent to the integral term. In the case of PbS-G QDs 
nanocomposite, a maximum specific capacity of $934.6 \mathrm{C} \mathrm{g}^{-1}$ is achieved at a scan rate of $5 \mathrm{mV} \mathrm{s}^{-1}$ which is significantly higher compared to the value of $681.5 \mathrm{C} \mathrm{g}^{-1}$ of bare PbS QDs at the same scan rate.

\subsubsection{Galvanostatic charge discharge (GCD)}

The GCD plots were analyzed for the fabricated electrodes at various current densities (2 to $\left.12 \mathrm{~A} \mathrm{~g} \mathrm{~g}^{-1}\right)$ in the potential window range of $0 \mathrm{~V}$ to $0.39 \mathrm{~V}$ and are shown in Fig. $8(\mathrm{a}, \mathrm{b})$. Nonlinearity of the discharge curves as can be seen from Fig. 8 (a,b) reveals that bare PbS QDs and PbS-G QDs nanocomposite exhibit battery type behavior. However, PbS-G QDs nanocomposite exhibits longer discharge time compared to bare PbS QDs (Fig. 8b). The increase in discharge time of the PbS-G QDs nanocomposite in due to the presence of GQDs, which were able to extend the duration of energy dissipation time due to large interfacial area accessible for the electrons for Faradaic reactions [50]. The specific capacity (Qs) of the materials was calculated from GCD plots using the following relation (3) [51]

$$
Q_{S}=\frac{I}{m}\left(\frac{\Delta t}{3.6}\right) \quad \mathrm{C} \mathrm{g}^{-1}
$$

where, $\mathrm{I}$ is the discharge current (A), $\Delta \mathrm{t}$ is the discharge time (s) and $\mathrm{m}$ is the mass $(\mathrm{g})$ of the active material. The calculated specific capacity and specific capacitance of bare PbS QD and PbS-G QDs nanocomposite at various current densities are shown in Fig.8c. As can be seen from Fig.8c, at current density of $2 \mathrm{~A} \mathrm{~g}^{-1}$ the specific capacities were $261.4 \mathrm{C} \mathrm{g}^{-1}$ and $577.9 \mathrm{C} \mathrm{g}^{-1}$ for bare PbS QD and PbS-G QDs nanocomposite, respectively. Also, the specific capacitance and specific capacity decreased with current density in both the cases. At lower current density, the electrolyte ions have sufficient time to reach the active sites of the electrode surface which resulted in high specific capacity, whereas the accessibility of the electrolyte ions towards electrode surface is restricted at high current densities resulting in decreased specific capacities. It is clear that PbS-G QDs nanocomposite exhibit high performance compared to $\mathrm{PbS}$ QDs. The introduction of GQDs into $\mathrm{PbS}$ QDs increased the rate capability which may be due to high dispersion and the intercalation of $\mathrm{PbS}$ QDs onto the GQDs networks which boosted the electrochemical performance of PbS-G QDs nanocomposite. 

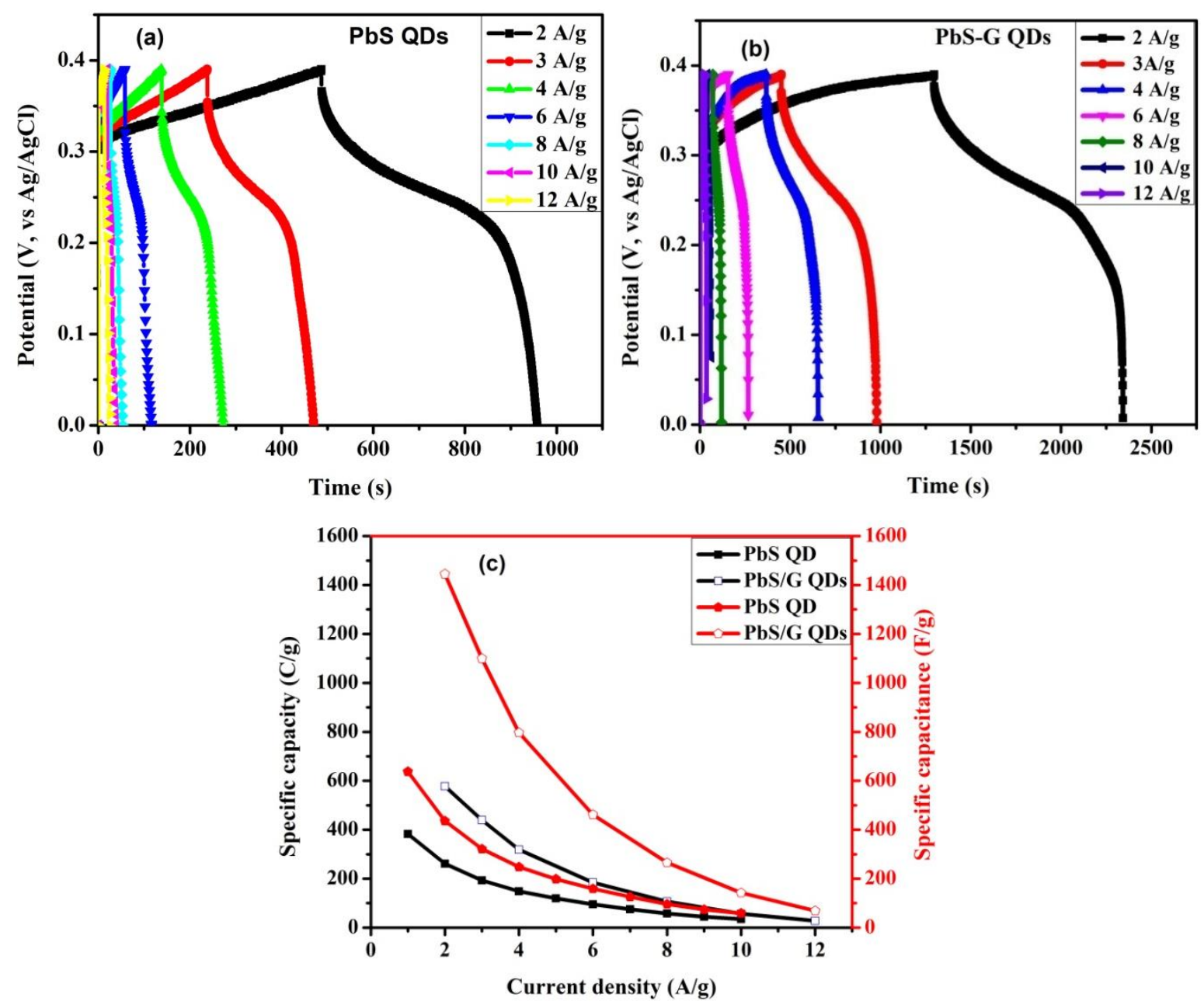

Fig. 8. Charge and discharge curves of (a) bare PbS QDs, (b) PbS-G QDs nanocomposite at various current densities and (c) Specific capacity (left-block color) with Specific capacitance (Right-red colour) of bare $\mathrm{PbS}$ QDs and $\mathrm{PbS}-\mathrm{G}$ QDs nanocomposite at different current densities

\subsubsection{Electrochemical impedance spectroscopy (EIS)}

To understand the intrinsic mechanism occurring on the electrodes, EIS studies was performed by measuring the resistance of charge transfer $\left(R_{c t}\right)$ and the frequency range of 0.1 to $100 \mathrm{kHz}$ at an amplitude of $10 \mathrm{mV}$. In the high frequency region of the complex plane impedance plot (Nyquist plot) the $\mathrm{R}_{\mathrm{ct}}$ is acquired from the diameter of the semicircle describing the charge transfer resistance occurring at the interface of electrode/electrolyte [52, 53]. From Fig. 9, bare PbS QDs shows large semicircle with $\mathrm{R}_{\mathrm{ct}}$ value of $7.74 \Omega$. Large semicircle demonstrates that PbS QDs has high interfacial resistance with poor behaviour of charge propagation. The diameter of the semicircle reduces significantly after GQD's is introduced into PbS QDs, with $\mathrm{R}_{\mathrm{ct}}$ value of $1.14 \Omega$, indicating that $\mathrm{PbS}-\mathrm{G}$ QDs nanocomposites has better charge transport behaviour and low interfacial resistance in comparison to bare $\mathrm{PbS}$ QDs. Equivalence series resistance (ESR) is obtained from the intercept on the real axis at the high frequency region of the Nyquist plot which is 
related to the internal resistance of the electrode and the total solution resistance. The ESR value of $1.41 \Omega$ for PbS-G QDs nanocomposite is lower in comparison to $1.59 \Omega$ of bare PbS QDs.

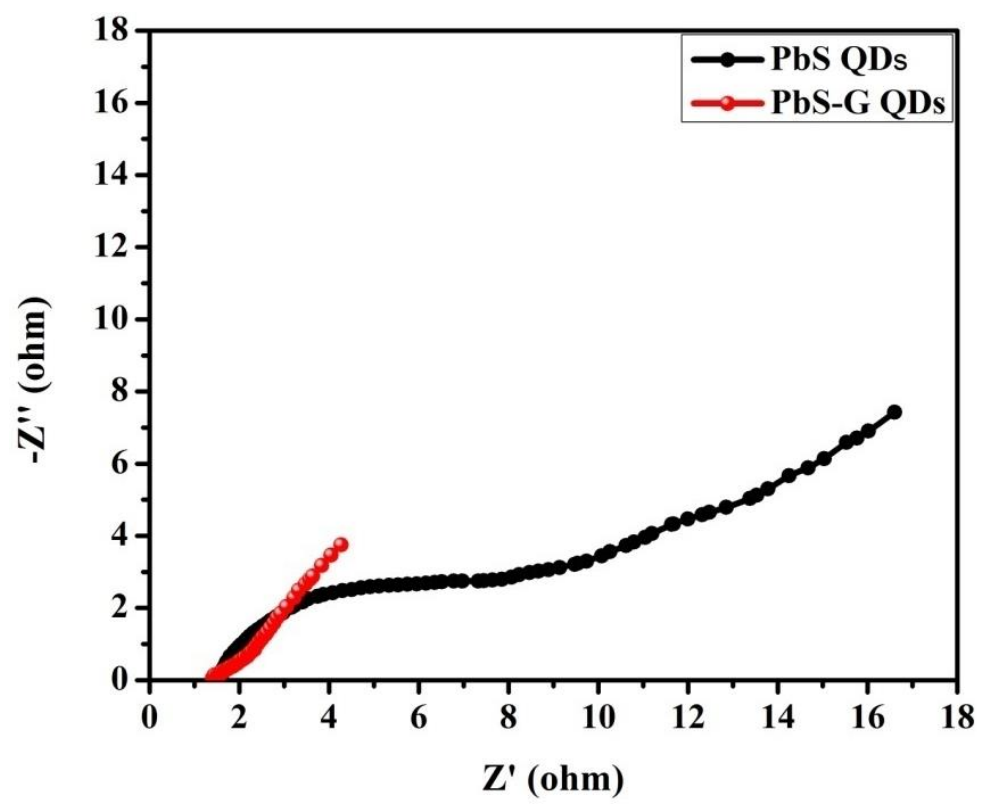

Fig.9. Nyquist plots of bare $\mathrm{PbS}$ QDs and $\mathrm{PbS}-\mathrm{G}$ QDs nanocomposite.

The energy (E) and power $(\mathrm{P})$ densities are the prime parameters to evaluate the functioning of energy storage devices. For evaluation of both the parameters, following relations have been exploited $[54,55]$

$$
\begin{aligned}
& E=\frac{1}{2 \times 3.6} Q_{s}(\Delta t)^{2} \\
& P=\frac{E \times 3600}{T}
\end{aligned}
$$

The fabricated supercapattery with $\mathrm{PbS}-\mathrm{G}$ QDs nanocomposite stored relatively higher energy and power densities of $166 \mathrm{Wh} \mathrm{kg}^{-1}$ and $576 \mathrm{~W} \mathrm{~kg}^{-1}$ respectively at a current density of $2 \mathrm{~A} \mathrm{~g}^{-1}$ compared to bare $\mathrm{PbS}$ QDs, which exhibited energy and power densities of $110 \mathrm{Wh} \mathrm{kg}^{-1}$ and $250 \mathrm{~W} \mathrm{~kg}^{-1}$ at the same current density. However, the value of energy density gradually decreased with a significant increase in the power density of bare $\mathrm{PbS}$ QDs and PbS-G QDs nanocomposite at higher current densities as shown in Fig. 10(a). The cyclic stability evaluation of supercapattery is an important parameter for the galvanostatic charging/discharging process at a current density of $2 \mathrm{~A} \mathrm{~g}^{-1}$. The high cyclic stability is observed for PbS-G QDs nanocomposite based supercapattery at a potential window range of 0 to $0.39 \mathrm{~V}$ for 5000 cycles as shown in Fig. 10b. Furthermore, PbS-G QDs nanocomposite showed high-capacity retention of $97.4 \%$ after 5000 cycling operation compared to 
bare $\mathrm{PbS}$ QDs (94.1\%) due to the excellent reversibility of the carbon materials (graphene) in PbSG QDs. The experimental results demonstrated that PbS-G QDs nanocomposite exhibits high specific capacity, high energy density with power density and excellent cyclic stability which makes it suitable for device applications. The accompaniment of carbon material (GQDs) with these pseudocapacitive materials further improves the capacity, energy density and also the electrochemical stability of PbS-G QDs nanocomposite.
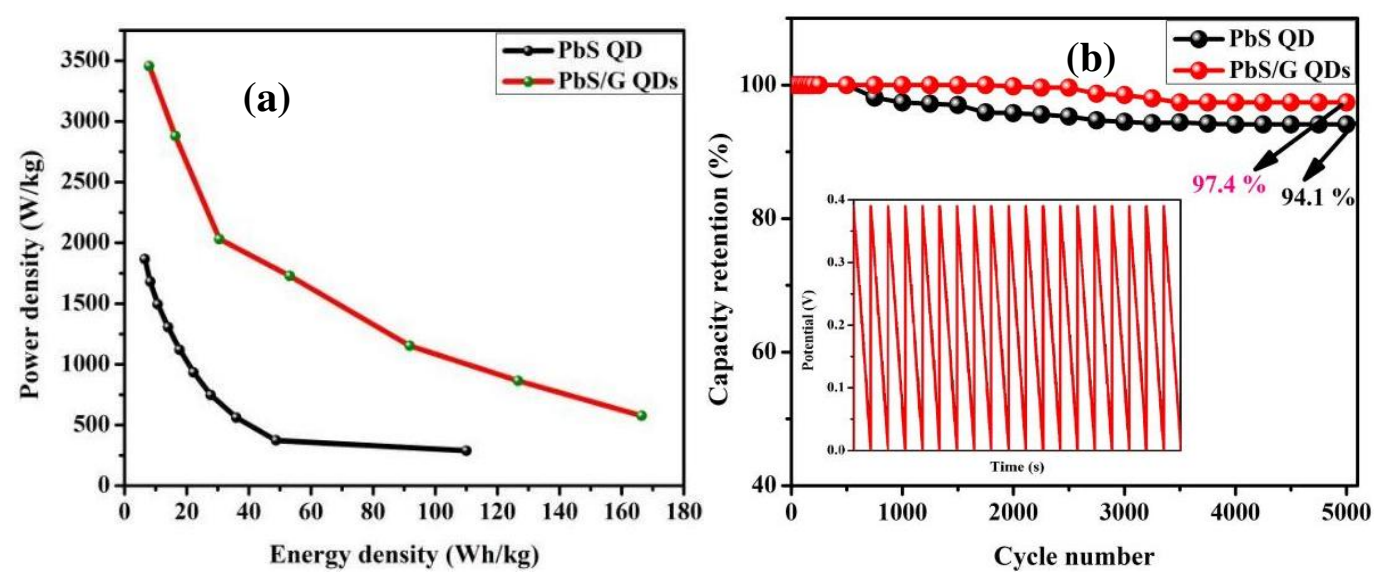

Fig.10. (a) The Ragone plots of bare PbS QDs and PbS-G QDs nanocomposite. (b) Cycling performance at $2 \mathrm{~A} \mathrm{~g}^{-1}$ and inset shows the last ten cycles.

\section{Conclusions}

Bare PbS QDs, GQD's and PbS-G QDs nanocomposite were prepared by simple processes of chemical bath method, ultrasonication method and solvothermal process respectively. The prepared samples were characterized by several analytical techniques to confirm the successful formation of PbS-G QDs nanocomposite and uniform distribution of PbS QDs onto the GQDs matrix. Electrochemical studies revealed that the performance of PbS-G QDs nanocomposite was superior compared to bare PbS QDs in terms of specific capacity. The improvement was particularly due to the conducting platform provided by GQDs in the nanocomposite. Furthermore, supercapattery assembled with $\mathrm{PbS}-\mathrm{G}$ QDs nanocomposite electrode material achieved a high energy density and power density of $166.45 \mathrm{Wh} \mathrm{kg}^{-1}$ and $576.01 \mathrm{~W} \mathrm{~kg}^{-1}$ respectively compared to bare $\mathrm{PbS}$ QDs with energy density and power density of $48.76 \mathrm{Wh} \mathrm{kg}^{-1}$ and $373.24 \mathrm{~W} \mathrm{~kg}^{-1}$. The cyclic stability evaluation of PbS-G QDs nanocomposite showed its superior long-term stability with high-capacity retention of $97.4 \%$ compared to bare $\mathrm{PbS}(94.1 \%)$ after 5000 cycles. All these features make PbS-G QDs nanocomposite a superior material for supercapattery application. 


\section{REFERENCES}

1. A. Burke, Electrochim. Acta, 53, 1083-1091, (2007).

2. Brian Kihun Kim, Serubbable Sy, Aiping Yu, and Jinjun Zhang, Handbook of Clean Energy Systems, 1-25, (2015).

3. Z. S Wu, K. Parvez, X. Feng, K. Mü llen, Nat. Commun., 4, 2487-2494, (2013).

4. I. Dincer, Renew. Sustain. Energy Rev., 4, 157-175, (2000).

5. M. Winter, R.J. Brodd, Chem. Rev., 104(10), 4245-422270, (2004).

6. G. Wang, L. Zhang, J. Zhang, Chem. Soc. Rev., 41, 797-828, (2012).

7. G. Zhao et al., Nano Energy, 47, 547-555, (2018).

8. S. Najib, E. Erdem, Nanoscale Adv., 1, 2817-2827, (2019).

9. Z. Sun, X. Lu, Ind. Eng. Chem. Res, 51 (30), 9973-9979, (2012).

10. B.E. Conway, Journal of the Electrochemical Society, 138 (6), 1539-1548, (1991).

11. K. Naoi, P. Simon, The Electrochemical Society’s Interface, 17 (1), 34, (2008).

12. George Z. Chen, International Materials Reviews, 62, 4, 173-202, (2017).

13. C. Justin Raj, B.C. Kim, W.J. Cho, W.G. Lee, Y. Seo, K.H. Yu, J. Alloy. Compd., 586, 191196, (2014).

14. Z. Jiang, W. Lu, Z. Li, K.H. Ho, X. Li, X. Jiao, J.D. Chen, Mater. Chem. A, 2, 8603-8606, (2014).

15. H. Chauhan, M.K. Singh, S.A. Hashmi, S. Deka, RSC Adv., 5, 17228-17235, (2015).

16. Ikkurthi kanaka Durga, S.Srinivasa Rao, Jin-woo Ahn, Tae-yong Park, Bak Jin-Soo, Cho-In Ho, K.Prabakar, Hee-Je Kim, energies, 11, 1624, (2018).

17. C. Wei, C. Cheng, J. Zhao, Y. Wang, Y. Cheng, Y. Xu, W. Du, H. Pang, Chem. Asian J., 10, 679-686, (2015).

18. R. Ramachandran, M. Saranya, P. Kollu, B.P.C. Raghupathy, S.K. Jeong, A.N. Grace, Electrochim. Acta, 178, 647-657, (2015).

19. J. Tian, T. Shen, X. Liu, C. Fei, L. Lv, G. Cao, Sci. Rep., 6, 23094, (2016).

20. Zhao, D., Huang, J., Qin, R., Yang, G. \& Yu, J. Adv. Opt. Mater., 6, 1800979, (2018).

21. A.G. Shulga, L, Piveteau, S.Z. Bisri, M.V. Kovalenko, M.A. Loi, Adv. Electron. Mater., 2, 1500467, (2016).

22. Z. Yang, et al., ACS Nano, 9, 12327-12333, (2015). 
23. Elochukwu Stephen Agudosi, Ezzat Chan Abdullah, Arshid Numan, Nabisab Mujawar Mubarak, Siti Rahmah Aid, Raúl Benages Vilau, Pedro Gómez Romero, Mohammad Khalid \& Nurizan Omar, Scientific Reports, 10, 11214, (2020).

24. Q. Cheng, J. Tang, J. Ma , H. Zhang, N. Shinya , L.C. Qin, carbon, 49, 2917-2925, (2011).

25. T. Lu, L. Pan, H. Li , G. Zhu, T. Lv, X. Liu, Z. Sun, T. Chen, D.H.C. Chua, J. Alloys and Compounds, 509, 5488-5492, (2011).

26. J.S. Bonso, A. Rahy, S.D. Perera, N. Nour, O. Seitz , Y.J. Chabal, J.Power Sources,203, 227232, (2012).

27. Chencheng Sun, Mingze Ma, Jun Yang, Yufei Zhang, Peng Chen, Wei Huang \& Xiaochen Dong, Scientific reports, 4, 7054, (2014).

28. M. Jayalakshmi, M.M. Rao, J. Power Sources, 157, 624-629, (2006).

29. Ting Zhu, Baoyu Xia, Liang Zhouand Xiong Wen (David) Lou, J. Mater. Chem., 22, 7851-7855, (2012).

30. Chia-Ying Chen, Zih-Yu Shih, Zusing Yang, Huan-Tsung Chang, J. Power Sources, 215, 4347, (2012).

31. Y. Zou, Y. Wang, ACS Nano, 5, 8108-8114, (2011).

32. A. Magrini, S. Lazzari, L. Marenco, G. Guazzi, International Journal of Heat and Technology, 35(4), 689-699, (2017).

33. W. Hummers, R. Offeman, Preparation of graphitic oxide. J Am Chem Soc., 80, 1339-9, (1958).

34. A. Rosy, G. Kalpana, current applied physics, 18(9), 1026-1033, (2018).

35. Tianju Fan, Wenjin Zeng, Wei Tang, Chunqiu Yuan, Songzhao Tong, Kaiyu Cai, Yidong Liu, Wei Huang, Yong Min and Arthur J Epstein, Nanoscale Research Letters, 10, 55, (2015).

36. M. Saranya, G. Srishti, I. Singh, R. Ramachandran, C. Santhosh, C. Harish, T.M. Vanchinathan, M. BhanuChandra, A.N. Grace, Nanosci. Nanotechnol. Lett., 5, 349-355, (2013).

37. R. Das, S.S. Nath, R. Bhattacharjee, phy. E low dimens.syst. nanostruct, 43, 224-227, (2010).

38. J.C Tauc, Elsevier, Amsterdam, North - Holland, 23, 1034, (1972).

39. Hyun Jeong, Jung Hoon Song, Sohee Jeong \& Won Seok Chang, scientific report, 10, 12475 , (2020).

40. Tuinstra F and Koenig J L, J. Chem. Phys., 53, 1126, (1970).

41. K. Kneipp, H. Kneipp, I. Itzkan, R. R. Dasari, M. S. Feld, Phy. Rev. Lett., 78, 1667, (1997).

42. Chen, G. Z., Int. Mater. Rev., 62, 173-202, (2017).

43. J. Ji, L.L. Zhang, H. Ji, Y. Li, X. Zhao, X. Bai, X. Fan, F. Zhang, R.S. Ruoff, ACS Nano, 7, 6237-6243, (2013). 
44. S.S. Rao, I.K. Durga, N, Kundakarla, D. Punnoose, C.V.V.M. Gopi, A.E. Reddy, M. Jagadeesh, H.J. Kim, New J. Chem., 41, 10037-10047, (2017).

45. H. Pang, J. Deng, S. Wang, S. Li, J. Du, J. Chen, J. Zhang, RSC Adv., 2, 5930-5934, (2012).

46. C. Xiang, M. Li, M. Zhi, A. Manivannan, N. Wu, J. Power Sources, 226, 65-70, (2013).

47. K. Vijaya Sankar, S. Surendran, K. Pandi, M. Allin, V.D. Nithya, Y.S. Lee, R. Kalai Selvan, RSC Adv., 5, 27649-27656, (2015).

48. N. Duraisamy, Mater. Lett., 161, 694-697, (2015).

49. C. He, S. Sun, H. Peng, C.P. Tsui, D. Shi, X. Xie, Y. Yang, Compos. B Eng., 106, 81-87, (2016).

50. V. Augustyn, P. Simon, B. Dunn, Energy Environ. Sci., 7, 1597-1614, (2014).

51. P. Simon, Y. Gogotsi, B. Dunn, Sci. Mag., 343, 1210-1211, (2014).

52. J.E. Huang, X.H. Li, J.C. Xu, H.L. Li, Carbon, 41, 2731-2736, (2003).

53. P. Simon, Y. Gogotsi, Nat. Mater., 7, 845-854, (2008).

54. Q. Lu, M. W. Lattanzi, Y. Chen, X. Kou, W. Li, X. Fan, K.M. Unruh, J.G. Chen, J.Q. Xiao, Angew. Chem., Int. Ed., 50, 6847-6850, (2011).

55. G.S. Gund, D.P. Dubal, S.S. Shinde, C.D. Lokhande, ACS Appl. Mater. Interfaces, 6, 3176-3188, (2014). 
Figures
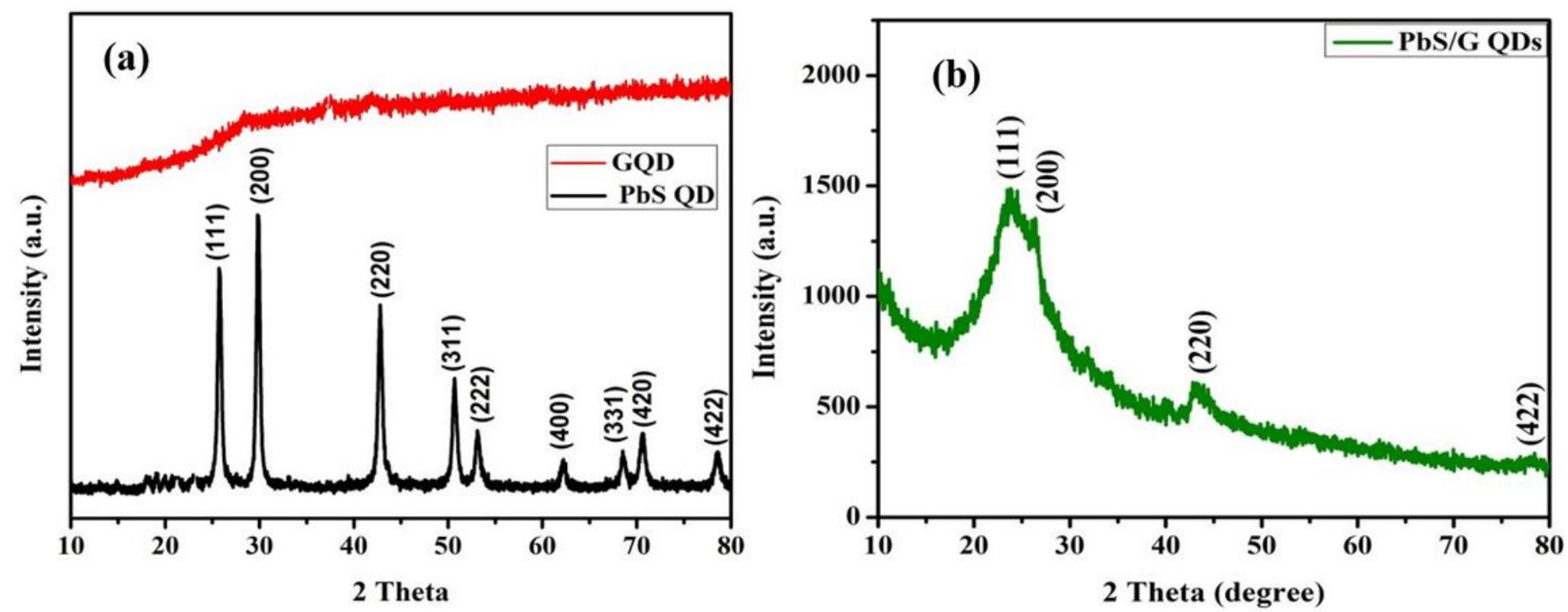

Figure 1

XRD patterns of the mesoporous (a) bare PbS QDs and GQDs, (b) PbS-G QDs nanocomposite.
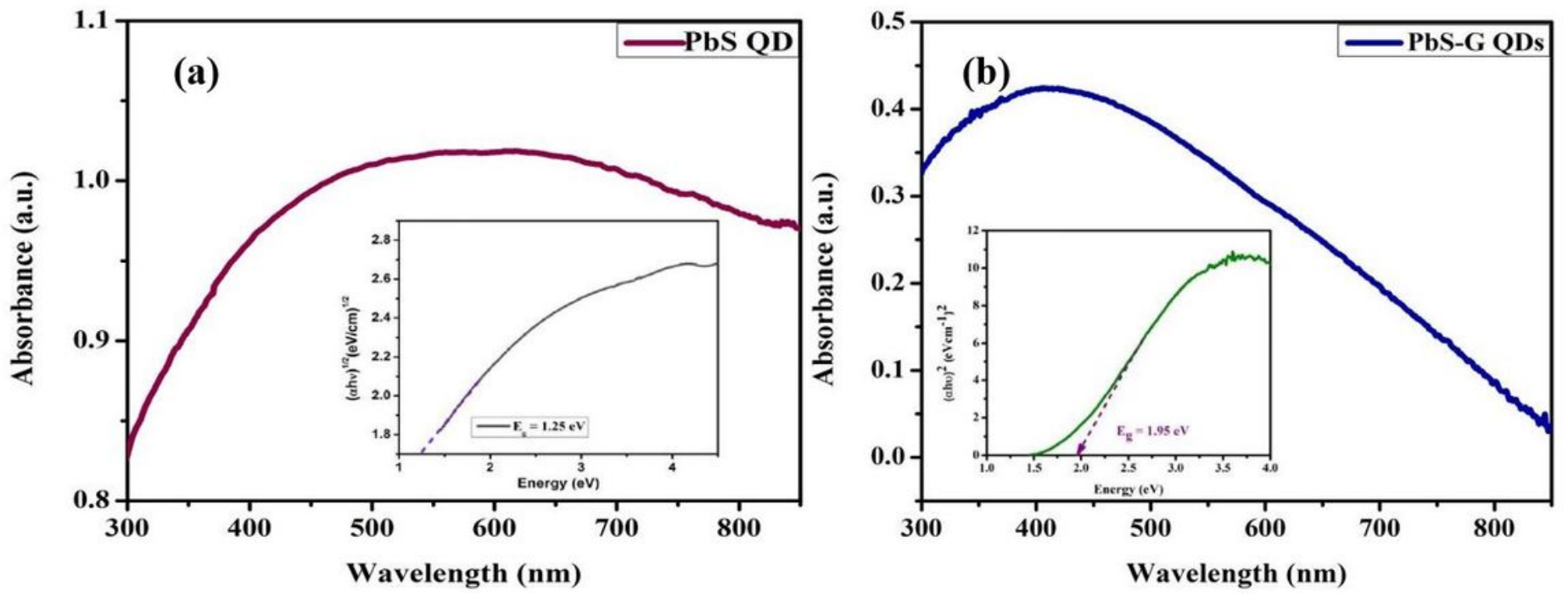

Figure 2

UV-vis DRS spectrum and optical energy gap diagram (inset picture) of (a) bare PbS QDs and (b) PbS-G QDs nanocomposite. 

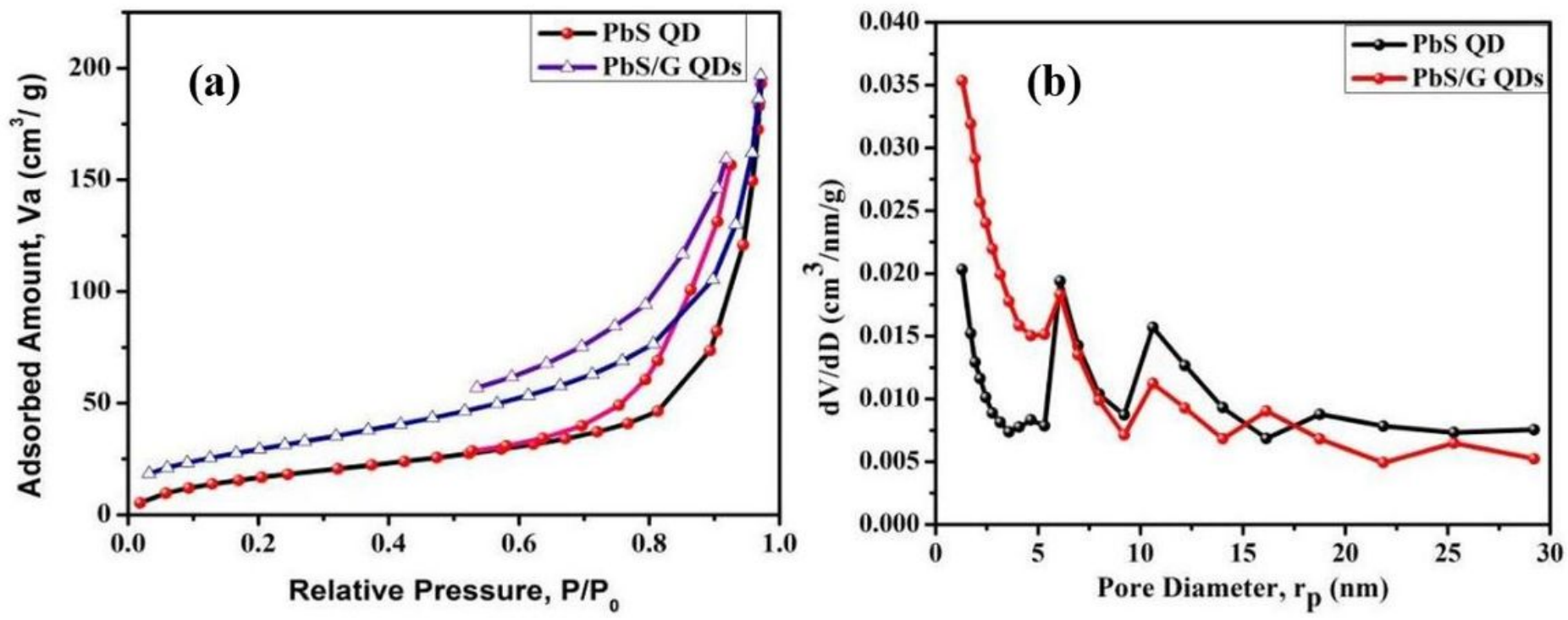

Figure 3

(a) The nitrogen adsorption/desorption isotherms and (b) pore size distributions for the bare PbS QDs and PbS-G QDs nanocomposites. 


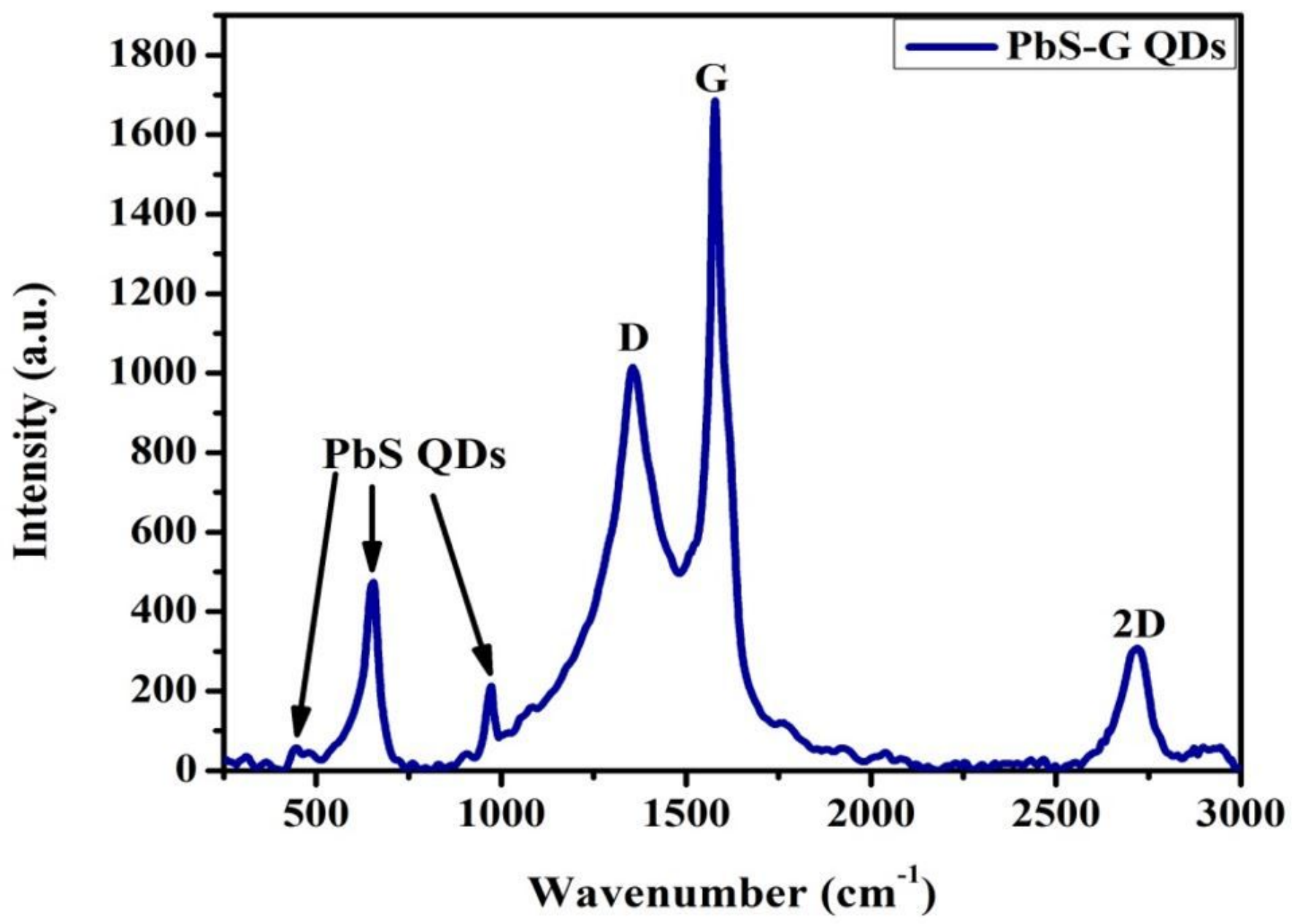

Figure 4

Raman spectrum of PbS-G QDs nanocomposite 

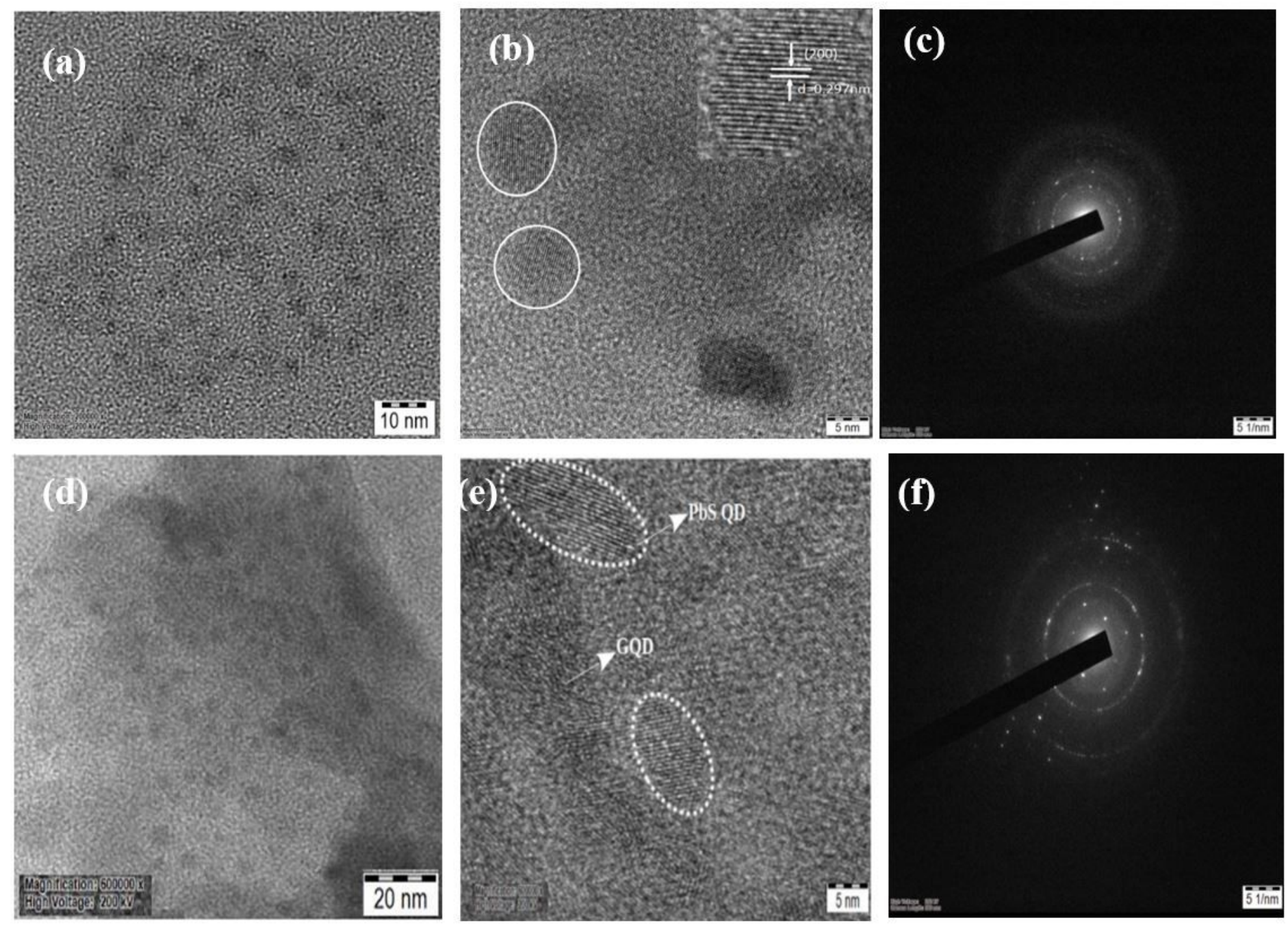

Figure 5

HRTEM images of (a \& b) bare PbS QD, (d \& e) PbS-G QDs nanocomposite and SAED patterns of (c) bare $\mathrm{PbS}$ QD and (f) PbS-G QDs. 

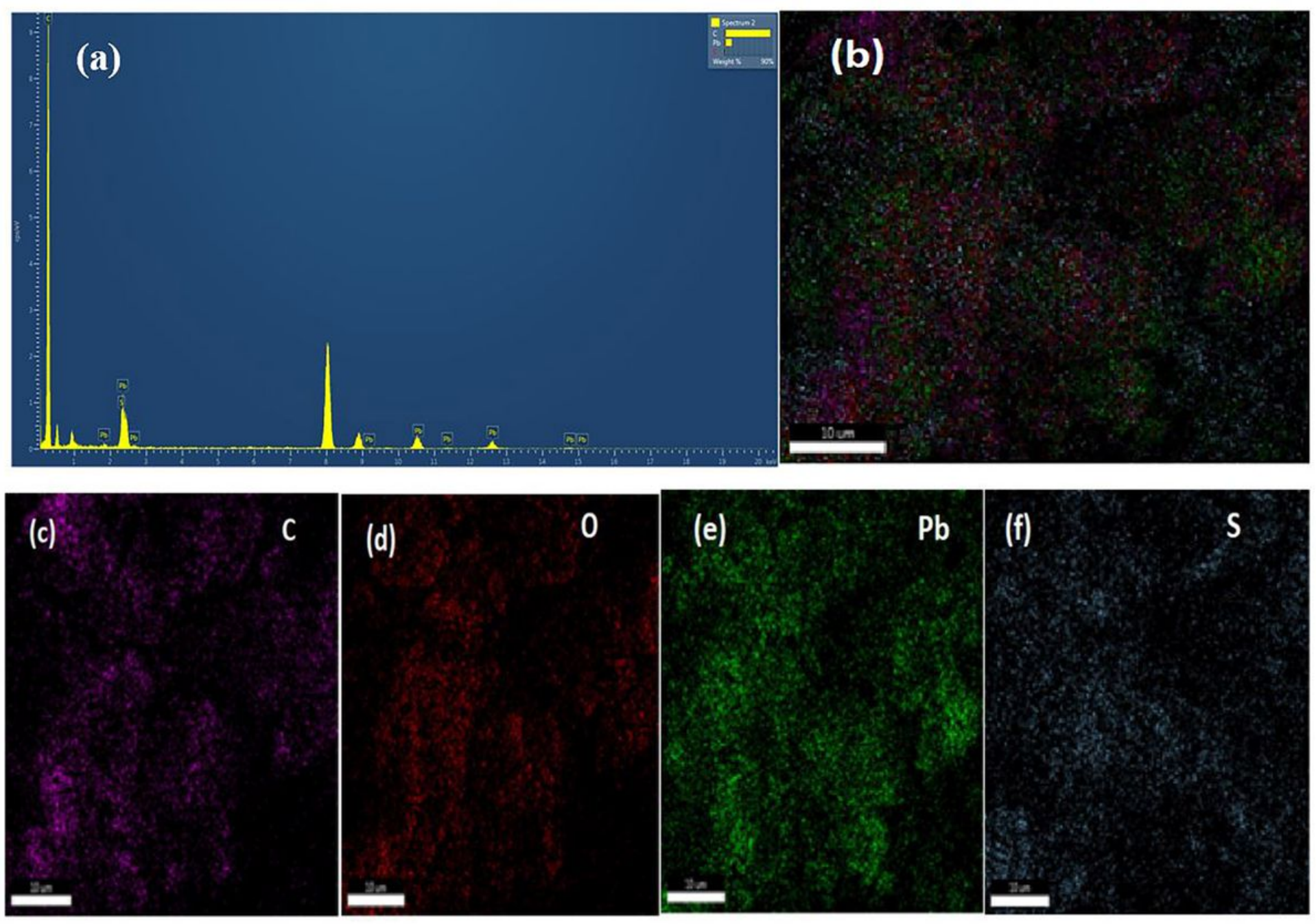

Figure 6

(a) EDX spectrum and mapping images of (b) PbS-G QDs nanocomposite, (c) C, (d) O, (e) Pb and (f) S in the nanocomposite. 

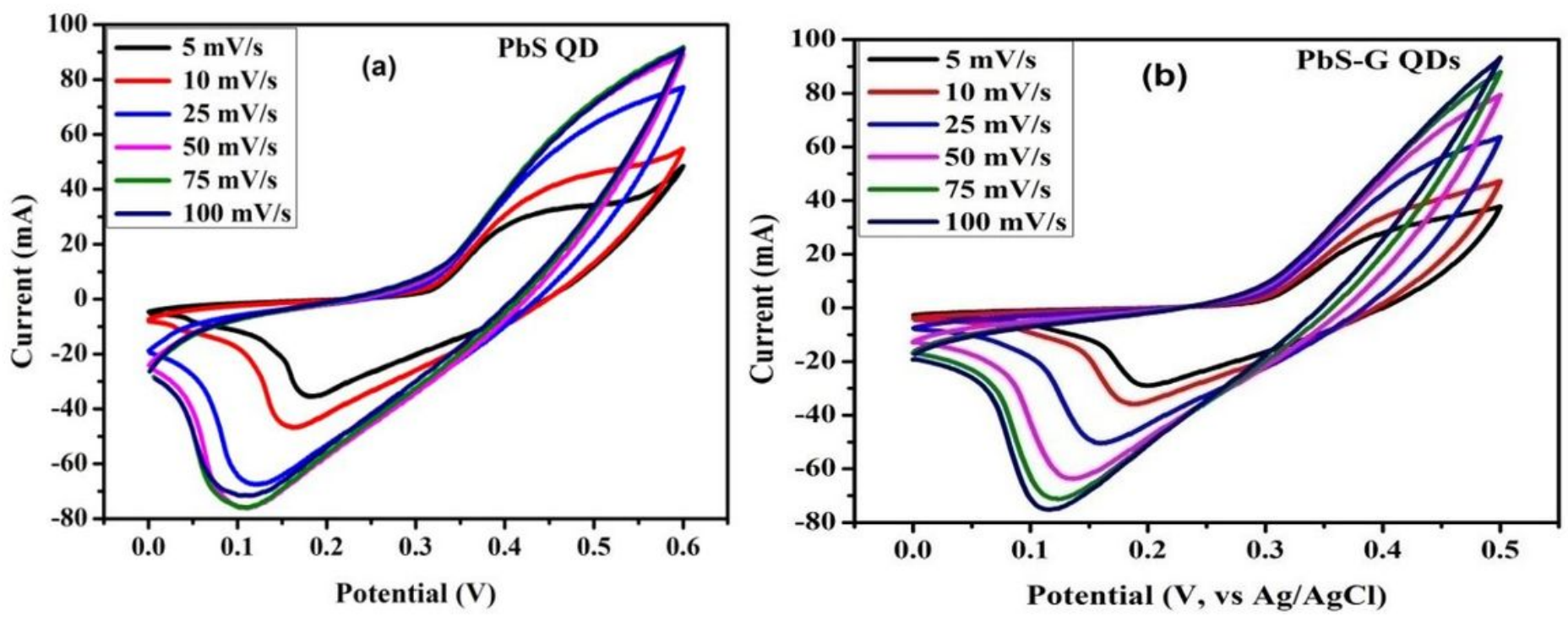

Figure 7

Cyclic voltammograms at different scan rates (a) bare PbS QDs and (b) PbS-G QDs nanocomposite 


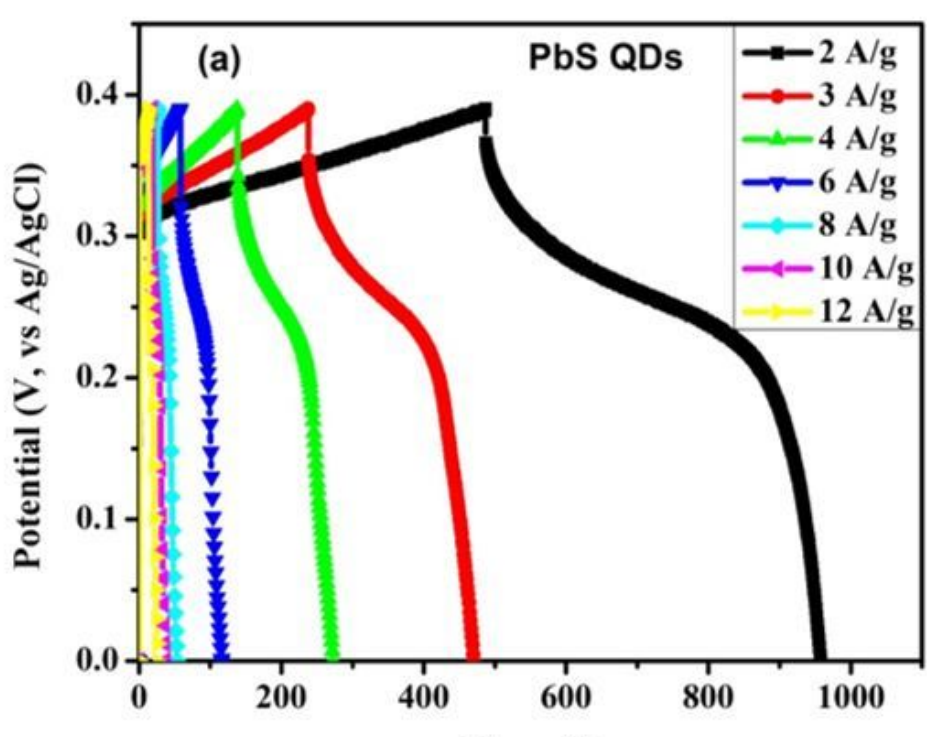

Time (s)

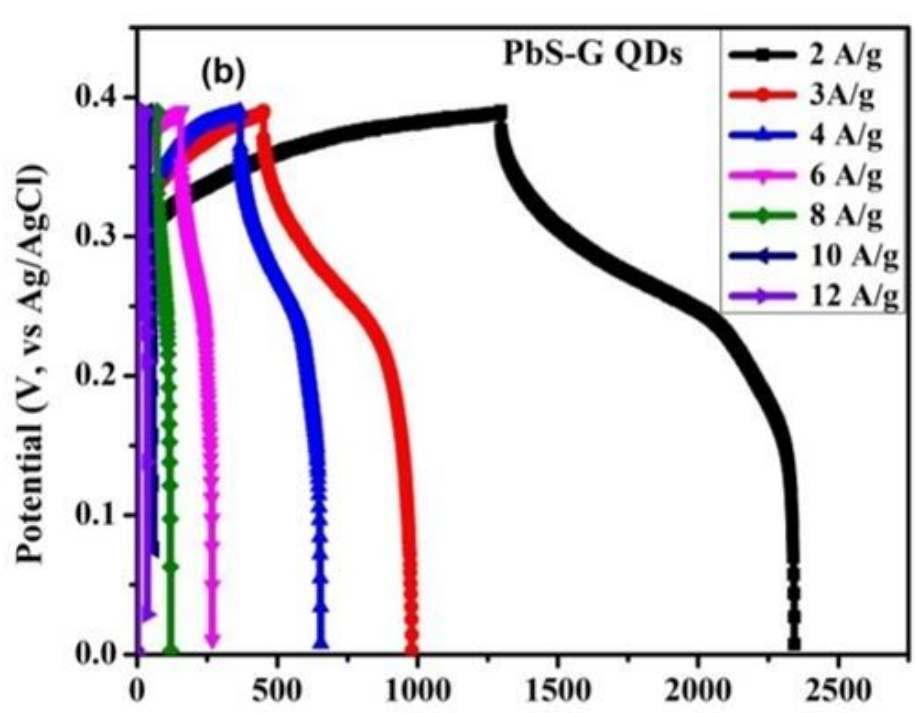

Time (s)

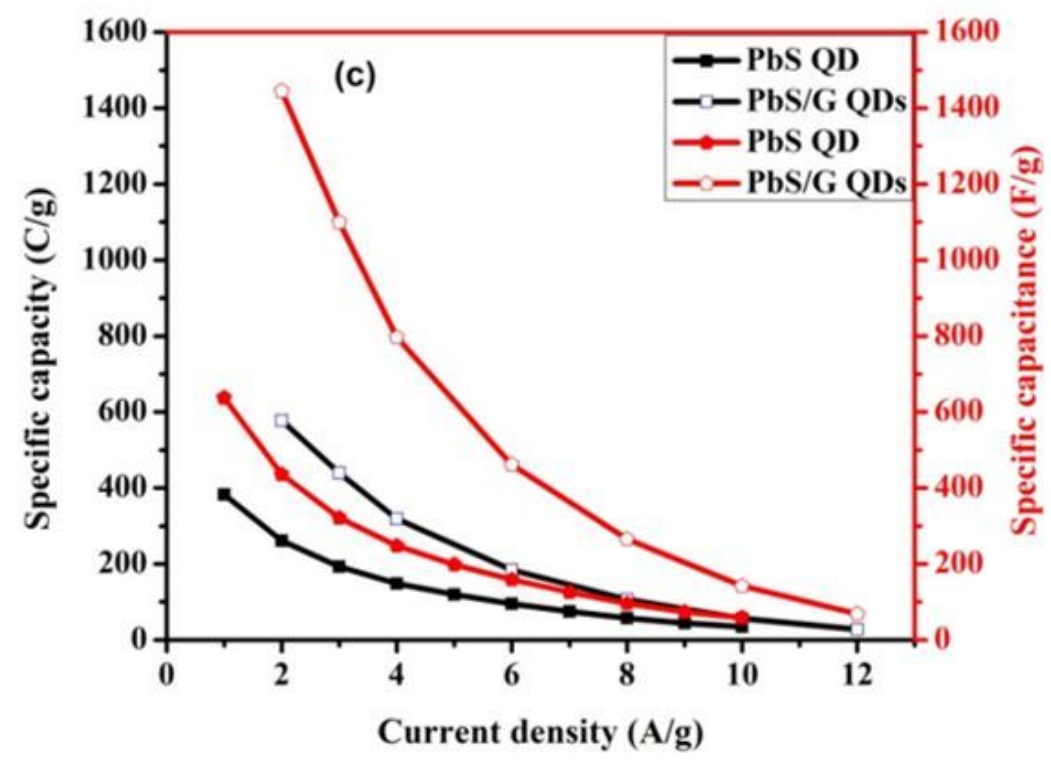

Figure 8

Charge and discharge curves of (a) bare PbS QDs, (b) PbS-G QDs nanocomposite at various current densities and (c) Specific capacity (left-block color) with Specific capacitance (Right-red colour) of bare PbS QDs and PbS-G QDs nanocomposite at different current densities 


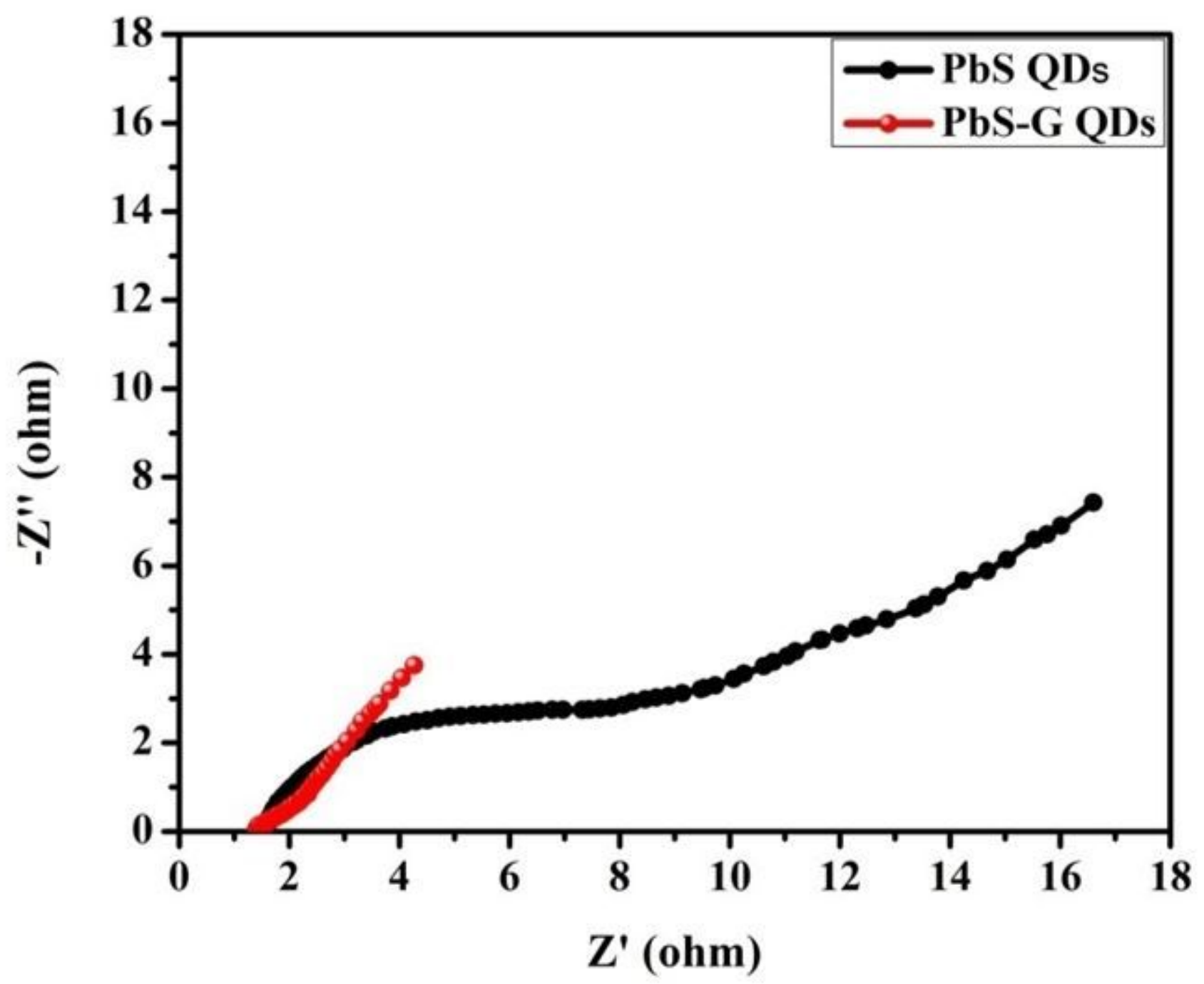

Figure 9

Nyquist plots of bare PbS QDs and PbS-G QDs nanocomposite. 

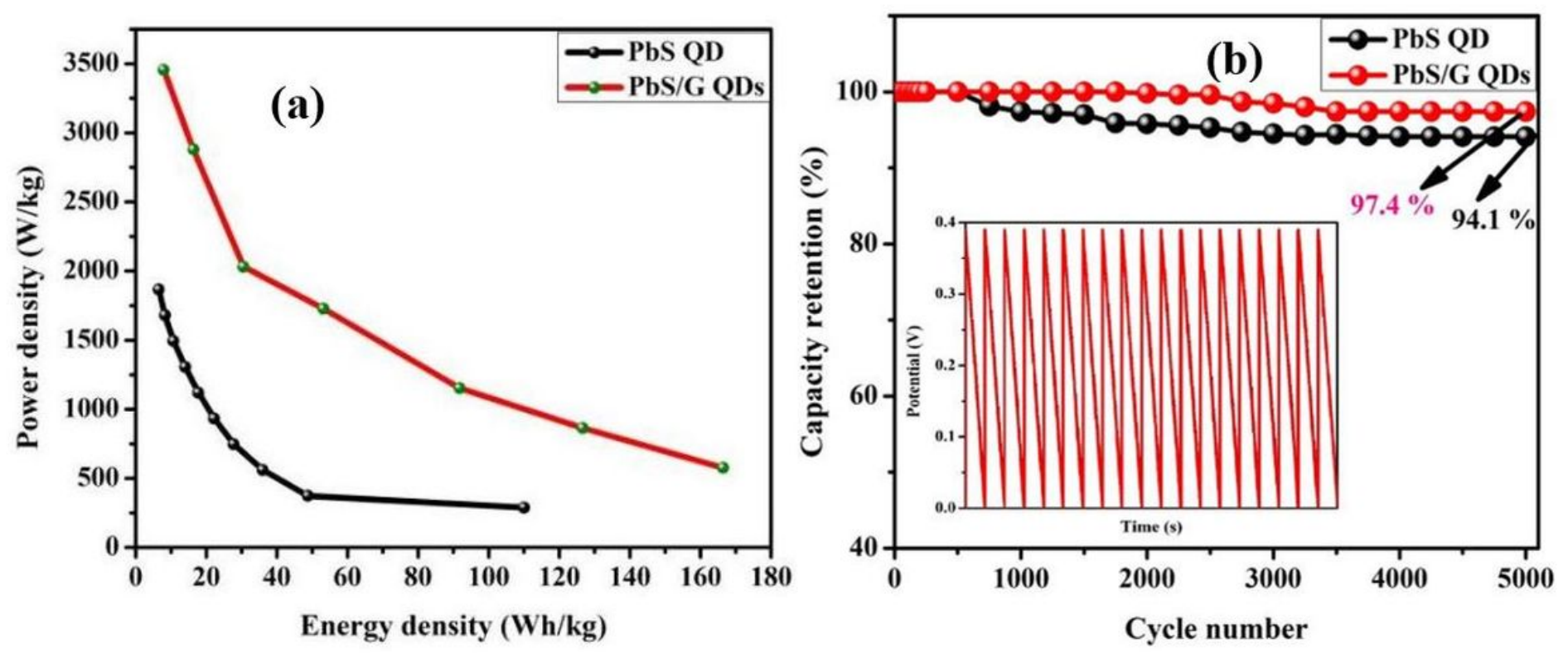

Figure 10

(a) The Ragone plots of bare PbS QDs and PbS-G QDs nanocomposite. (b) Cycling performance at $2 \mathrm{~A} \mathrm{~g}^{-1}$ and inset shows the last ten cycles. 